\title{
Barrières d'étanchéité dans les sites de décharges: développements récents
}

\section{W.F. VAN IMPE}

Vice-Président ISSMFE

Laboratoire de Mécanique

des Sols,

Université de Gand (RUG),

Université Catholique

de Louvain (KUL),

Louvain - Belgique

\section{A. BOUAZZA}

Chercheur NFWO,

Laboratoire de Mécanique

des Sols,

Université de Gand (RUG),

Gand-Belgique
Depuis le début des années 80 la mise en décharge des déchets est devenue un domaine particulièrement sensible. Ceci a conduit à la promulgation de nouvelles réglementations au sein de la Communauté européenne et internationale. La conséquence immédiate a été l'apparition d'une multitude de règlements régissant la construction des barrières d'étanchéités des sites de décharges. Ce texte passe en revue les récents développements dans ce domaine avec une attention toute particulière aux problèmes liés au transport des contaminants, sols non saturés et le contrôle de qualité de la barrière argileuse. Finalement un cas d'étude concernant l'utilisation d'un matelas bentonitique est présenté.

\section{Liners for waste disposal sites : recent developments}

new legislations in the European community and internationnaly which resulted in an array of regulations concerning landfill liner design. The present paper comments on the recent developments in liner design with a particular emphasis on problems linked to contaminant transport. unsaturated soils, and quality control of the compacted clay liner. Finally, a case study concerning the use of a geosynthetic clay liner (GCL) is presented. 


\section{Introduction}

Le progrès économique que la plupart des pays industrialisés ont connu dans le passé a engendré d'importants problèmes liés au stockage des déchets à la fois industriels et domestiques. Le stockage de toutes sortes de déchets allant des boues résiduelles toxiques aux déchets inertes a été fait d'une manière indiscriminatoire et anarchique dans des sites parfois non préparés pour les recevoir. La conséquence immédiate a été la pollution du milieu naturel avec des conséquences extrêmement variées, allant par exemple de la perte des vies humaines dans le cas de la pollution par le mercure le long de la baie de Minamata au Japon vers la fin des années 60 à la simple contamination des sols par des lixiviats de décharges municipales.

La sensibilisation aux problèmes associés à la protection de l'environnement, à travers les différents mouvements écologiques et les médias, a fait qu'une pression énorme a été exercée sur les pouvoirs publics. La dernière décennie a vu l'émergence de programme de nettoyage des sites pollués, des décharges sauvages (non contrôlées), des friches industrielles, etc., comme ceux identifiés par le programme américain Superfund et le répertoire des terres contaminées établi par la Communauté européenne.

La principale technique d'élimination des déchets est la mise en décharge qui reste d'ailleurs le procédé le plus utilisé par les collectivités locales. Aux États-Unis, $80 \%$ des déchets sont mis en décharge, $10 \%$ sont incinérés et $10 \%$ sont recyclés (Rebeiz et Mielich, 1995). Tandis que dans la Communauté européenne $70 \%$ des déchets ménagers et $35 \%$ des déchets industrieis sont stockés dans les centres d'enfouissement (Street, 1994). Les décharges ont été traditionnellement construites en admettant que le sol adjacent et les eaux souterraines étaient capables de diluer le jus des lixiviats et d'atténuer son effet.

En Grande-Bretagne, par exemple, les décharges étaient construites d'une manière délibérée sur des sites caractérisés par des perméabilités élevées. Il a été supposé que le mélange des lixiviats avec un large volume d'eau souterraine était suffisamment efficace pour réduire la concentration des produits contaminants à un niveau inoffensif (Potter et Yong, 1993). Récemment, Kovacic et al. (1993) ont présenté un cas concernant une décharge située à Zagreb (Croatie). Elle a été construite directement sur des matériaux alluvionnaires sans aucune barrière d'imperméablisation. En Allemagne, les décharges étaient traditionnellement protégées par une barrière argileuse. Cette protection

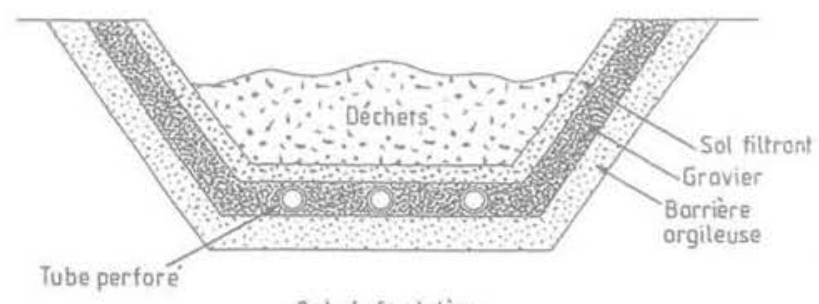

Sol de fondation

Fig. 1 Système d'étanchéisation avec barrière simple.

Single clay liner. peut être insuffisante; Bishop et Carter (1993) ont cité un cas où des métaux lourds avaient pénétré la barrière argileuse sur une certaine profondeur. Aux Etats-Unis, I'Agence de Protection de I'Environnement (EPA) estimait que $75 \%$ des décharges existantes ont causé une pollution des eaux souterraines. Jusqu'en 1982, la plupart des barrières d'imperméabilisation construites étaient argileuses (Fig. 1). Généralement, cette barrière est recouverte d'une couche drainante contenant des tubes perforés pour recueillir et évacuer les lixiviats. Cette couche est séparée des déchets solides par une couche de sol filtrante protégeant la perforation des tubes contre l'obstruction par des particules fines. En Suède, ce type de conception est toujours courant. Il existe approximativement 300 sites de décharges construits suivant cette configuration.

Plus récemment encore, Thomas et al. (1995) ont présenté un cas de contamination des sols survenu à Valdez (Alaska, USA) sous une barrière asphaltique surmontée d'une couche de moraine glaciaire dont l'épaisseur est de $0,9 \mathrm{~m}$. Cette contamination par une eau souillée de pétrole s'était étendue sur $2000 \mathrm{~m}^{2}$ avec une profondeur variant entre $3 \mathrm{~m}$ et $6 \mathrm{~m}$.

Dans les pays en voie de développement, la situation est catastrophique. Dans la plupart des décharges, les barrières d'étanchéisation et le système de collection et d'évacuation des lixiviats sont inexistants. L'explosion démographique et la concentration de la population dans les grandes villes et autour des zones industrielles font que les capacités des décharges sont dépassées d'une manière vertigineuse. Ceci est parfaitement illustré par la décharge de la «montagne fumante (smokey mountain) ) située à Manille (Philippines) et à un degré moindre par la décharge d'OuedSmar à Alger (Algérie) (Bouazza, 1993, 1993a) où la pollution de l'air et du sol a atteint un niveau sérieux.

Un autre aspect pénalisant est le coût du transport des déchets. En effet, plus la distance séparant la source et la décharge augmente, plus le coût augmente. Ce facteur fait que la décharge est localisée généralement non loin de la ville. La ville en expansion et dépassant les sites réservés aux déchets, cette situation entraîne la localisation des décharges dans des lieux indésirables. La ville de Mexico est un exemple typique de ce phénomène, avec une très vaste décharge au centre, complètement entourée par un développement urbain galopant. En Europe, ce phénomène est aussi existant notamment à Londres où l'extension de ses banlieues a atteint les vieilles décharges qui sont actuellement réaménagées.

\section{2}

\section{Sources de la contamination}

La pollution des eaux souterraines et des sols joue un rôle important dans l'évaluation des qualités de l'environnement dans les différentes localités. Il est reconnu, bien que le processus de pollution des sols soit lent et souvent sans conséquence immédiate et dramatique, qu'à long terme les effets de la pollution seront sérieux et parfois irréversibles. Plusieurs sources de contamination ont été identifiées et peuvent être classées en deux catégories :

- les activités liées au stockage des déchets et utilisant le sol comme récepteur de déchets; par exemple: décharge municipale, industrielle, décharge pour pro- 
duits toxiques et dangereux, bassin de décantation, etc.;

- les activités commerciales et industrielles pouvant entraîner accidentellement le rejet dans le sol d'une quantité importante de polluants. Ceci peut être le cas des fuites ou des déversements survenant lors du transport, de la manipulation, du stockage et de l'utilisation de différents produits à caractère polluant, dragage, déchets solides mis en décharges en solution.

D'autres sources peuvent être rajoutées, par exemple: les activités liées à l'agriculture où l'utilisation intentionnelle de produits chimiques engendrent une pollution des sols et des eaux.

Le tableau I donne d'une manière succincte le type de rejet, son volume, la concentration du contaminant et le type de facteur affectant la nature du rejet. Parmi tous les types de rejets énumérés dans le tableau I, la formation des lixiviats et le transport à travers le fond de la décharge ont été les plus étudiés.

Le lixiviat peut être défini comme un liquide qui a percolé à travers les déchets solides en extrayant des constituants des déchets sous une forme dissoute ou suspendue. Dans les décharges modernes, la formation des lixiviats est principalement causée par l'infiltration directe de l'eau météorique dans les déchets. En effet, Bonaparte (1995) spécifie que les teneurs d'autres sources de liquide sont relativement faibles pour induire la formation de lixiviats car:

1. Les règlements courants de construction de décharges prohibent le stockage des déchets liquides.
2. Les déchets solides municipaux et la plupart des autres types de déchets entreposés dans les décharges modernes ont de faibles teneurs en eau au moment du stockage.

3. Les règlements courants requièrent que les décharges soient construites de façon à prévenir l'infiltration des eaux de surface.

Dans les régions arides, la production de lixiviats peut être faible et parfois nulle. Keenan (1986) a indiqué que dans les régions où la pluviométrie est faible ( $300 \mathrm{~mm} / \mathrm{an}$ ) la formation de lixiviats était pratiquement nulle.

Les caractéristiques de lixiviats varient très largement d'une décharge à une autre. Cette variation est basée sur les propriétés des déchets, l'âge des déchets, la teneur en eau, la température, ainsi que d'autres facteurs. Les lixiviats sont classés comme des liquides aqueux ou des solutions coutenants des produits contaminants solubles dans l'eau, liquides non aqueux constitués de composants organiques non solubles dans l'eau ou un mélange des deux résultant de la formation d'un liquide biphasé. Shackelford (1994) donne une excellente classification des lixiviats formés dans les décharges. La décomposition des déchets génère aussi des gaz comme le méthane $\left(\mathrm{CH}_{4}\right)$ ou le dioxyde de carbone $\left(\mathrm{CO}_{2}\right)$. Ces gaz peuvent migrer à travers le sol et s'accumuler dans des espaces confinés pour produire des concentrations explosives, asphyxiantes ou toxiques. Ils peuvent aussi affecter la végétation existante autour de la décharge.

TARLEAUI Type de rejets polluants à la surface et sur l'eau.

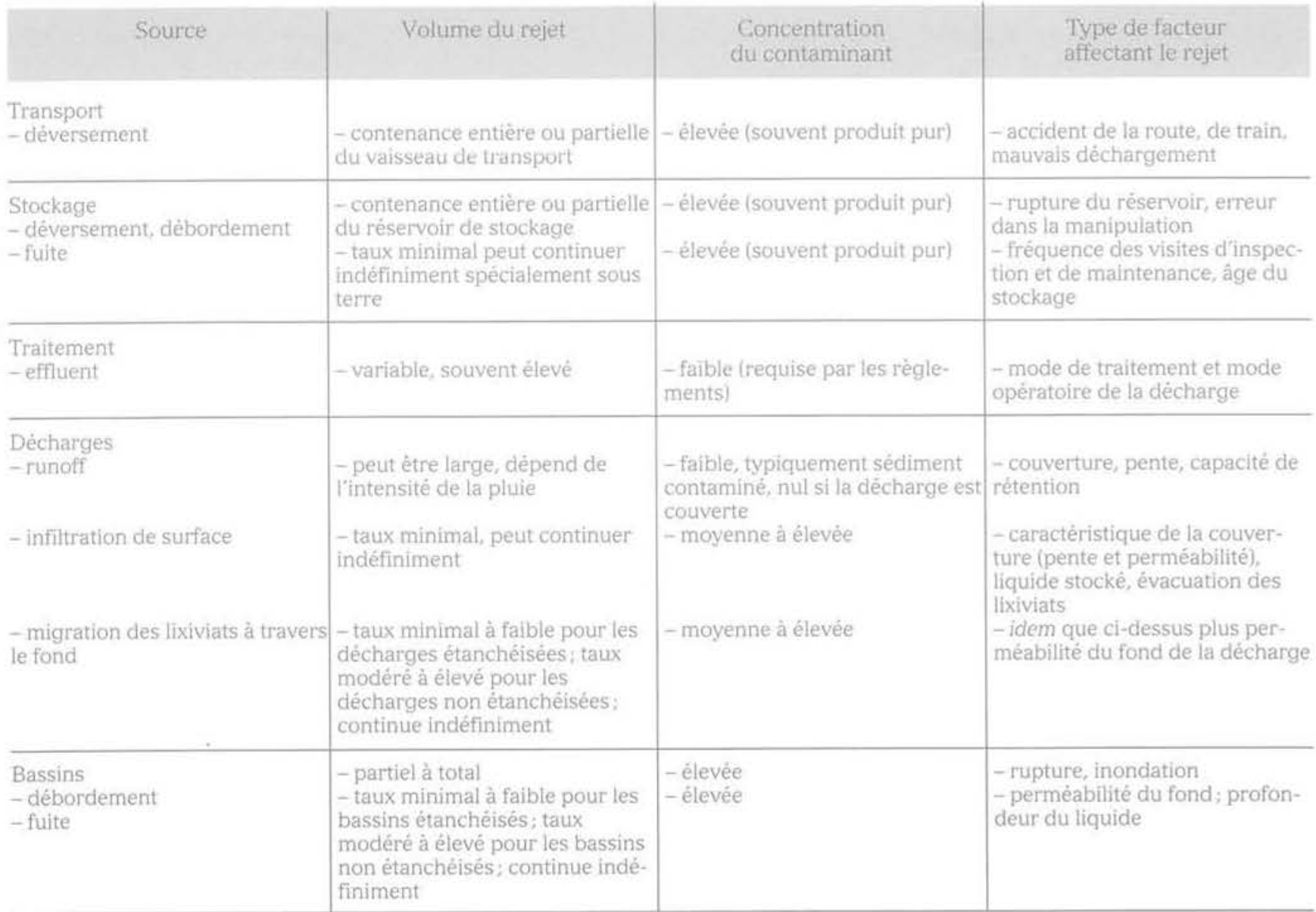


Il est à noter que le taux de production des gaz est une fonction de plusieurs facteurs tels que : la composition des déchets, le climat, la teneur en eau, la dimension des particules, le compactage, etc. Ce taux varie entre 0,12 et $0,41 \mathrm{~m}^{3}$ de gaz par kilo de déchets secs (Pohland et Harper, 1985). Ces gaz se composent de $40 \%$ à $60 \%$ de méthane, de dioxyde de carbone et de traces de gaz tel que le sulfure d'hydrogène. Un rapport émanant du comité de la qualité de l'air de l'État de Californie (Bennett, 1987) estime que l'émission de gaz (excluant le méthane) est de $35 \mathrm{~kg}$ par million de kilo de déchets.

\section{3}

\section{Transport des contaminants}

Dans la plupart des règlements régissant la conception des sites de décharges, la valeur minimale de la conductivité hydraulique est perçue comme le paramètre le plus important dans l'évaluation de leur imperméabilisation par une barrière argileuse ou autres.

Cependant, il est de plus en plus reconnu que ce standard minimal ne satisfait plus le but final qui est d'empêcher la migration des constituants des déchets à travers la barrière d'étanchéisation durant la vie d'une décharge donnée.

L'importance de la diffusion dans des sols peu perméables a été souvent occultée du fait que la plupart des travaux étaient orientés vers la satisfaction d'un critère minimal. Il a été montré que les constituants des lixiviats peuvent migrer à travers les barrières argileuses par diffusion moléculaire grâce à un gradient chimique.

Gillham et al. (1984) ont indiqué que la diffusion est un facteur dominant dans le processus de transport des contaminants quand la vitesse d'écoulement est approximativement égale à $0,005 \mathrm{~m} / \mathrm{an}$. Pour une barrière argileuse, la vitesse d'écoulement correspond généralement à une conductivité hydraulique saturée (k) de $8.10^{-11} \mathrm{~m} / \mathrm{s}$ (quand $\mathrm{n}=0,5$ et $\mathrm{i}=1$ ). Certains règlements spécifient une valeur minimale de $\mathrm{k} \leq 1.10^{-9} \mathrm{~m} / \mathrm{s}$, dans ce cas la diffusion peut être un facteur significatif, sinon dominant, dans le processus de transport. Reades et al. (1990) ont présenté le cas de décharge de la vallée de Keele, située dans l'état du Toronto (Canada) oủ l'effet de la diffusion dans le transport des constituants des lixiviats à travers une barrière argileuse a été clairement démontré (Fig. 2). Cette barrière a été construite de façon à avoir une valeur minimale de $k$ égale à $1.10^{-10} \mathrm{~m} / \mathrm{s}$. Après quatre ans de fonctionnement, du chlorure a été détecté à une profondeur de $0,4 \mathrm{~m}$ dans la barrière argileuse dont l'épaisseur était de $1,2 \mathrm{~m}$. Un calcul rapide basé sur le transport advectif (loi de Darcy) oủ le temps de migration requis par une solution non réactive comme le chlorure pour traverser un sol saturé d'épaisseur (L) est donné par (Van Impe et Bouazza, 1995):

où :

$$
\mathrm{t}=\mathrm{L} / \mathrm{v}_{\mathrm{s}}=\mathrm{Ln} / \mathrm{k}_{1}
$$

$t=$ temps de transit de la solution;

$\mathrm{n}=$ porosité de la barrière;

$\mathrm{k}=$ conductivité hydraulique de la barrière;

$\mathrm{i}=$ gradient hydraulique ;

$\mathrm{L}=$ épaisseur de sol considéré $(0,4 \mathrm{~m})$.

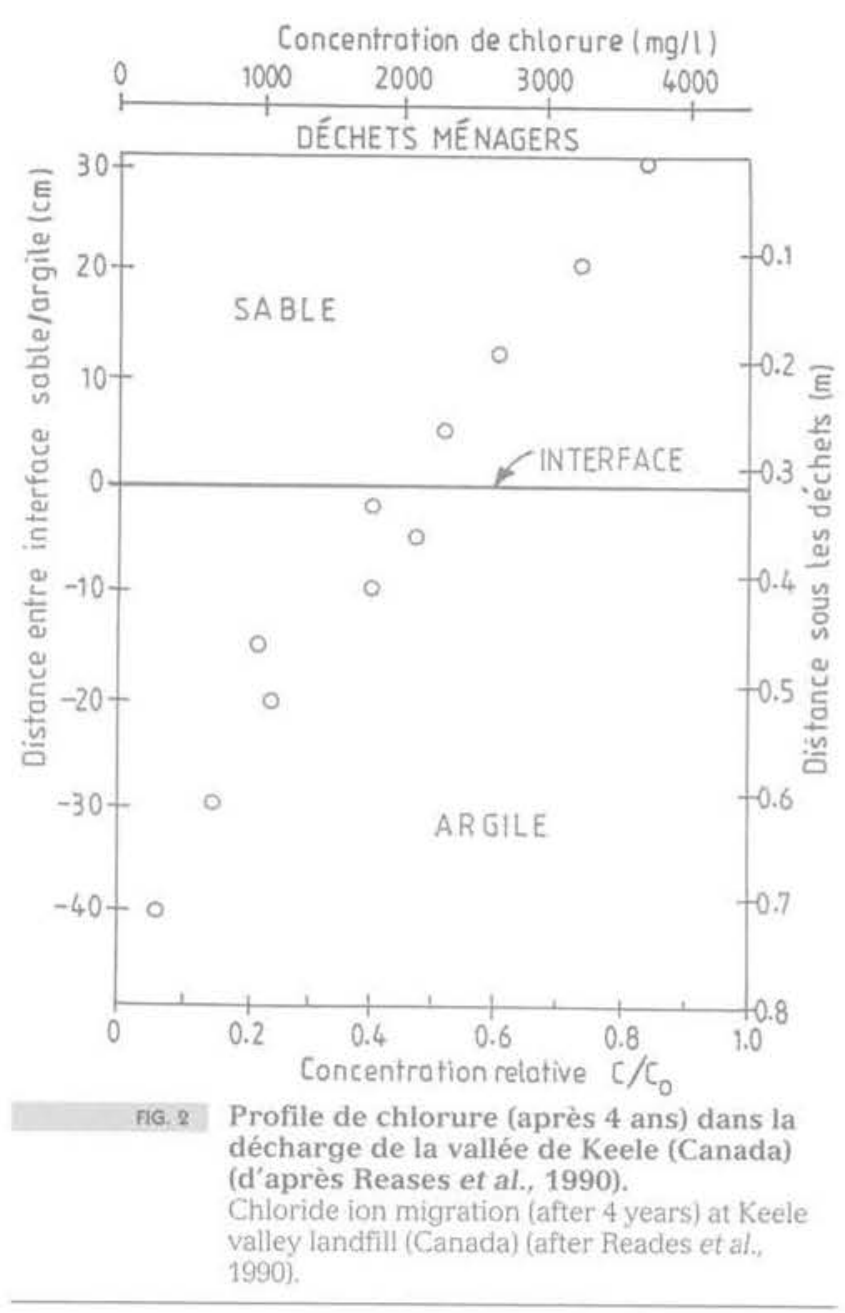

Si on suppose que $\mathrm{n}=0,4$ et $\mathrm{i}=1,5$ alors le temps requis pour atteindre la profondeur de $0,4 \mathrm{~m}$ est égal à 34 ans. Cette durée de transit est très supérieure à la valeur mesurée par Reades et al. (1990).

Le cas présenté ci-dessus et d'autres cas cités dans la littérature (Goodall et Quigley 1977, Crooks et Quigley 1984, Johnson et al. 1989, Barbosa et al. 1995) prouve la nécessité de considérer le transport diffusif quand on évalue les mouvements des lixiviats à travers une barrière argileuse.

\section{1}

\section{Aspect théorique}

La théorie qui régit le phénomène présenté dans la section précédente est basée sur l'équation d'advection et de dispersion qui décrit le transport unidimensionnel transitoire d'une solution donnée à travers un sol homogène (Freeze et Cherry, 1979). Cette équation s'écrit:

$$
\frac{\partial C_{r}}{\partial t}=\frac{D}{R_{d}}\left(\frac{\partial^{2} C_{r}}{\partial x^{2}}\right)-\frac{v}{R_{d}}\left(\frac{\partial C_{r}}{\partial x}\right)
$$

où $\mathrm{D}$ est le coefficient de dispersion hydrodynamique: $\mathrm{C}_{\mathrm{r}}=$ concentration de la solution dans l'eau interstitielle du sol; $R_{d}=$ facteur de retardement; $t=$ temps; $x=$ distance macroscopique dans la direction de l'écoulement; et $\mathrm{v}=$ vitesse d'écoulement donnée par la loi de Darcy. 
Le facteur de retardement $R_{d}$, prend en compte l'équilibre instantané, réversible et linéaire de l'adsorption des solutions réactives. Dans le cas des solutions réactives (adsorbantes) : $R_{d}>1$; pour le cas des solutions non réactives (non adsorbantes): $R_{\mathrm{d}}=1$. Cette valeur de $R_{d}$ est donnée avec l'hypothèse que l'écoulement se fait à travers tous les espaces poreux.

Le coefficient de dispersion hydrodynamique est la somme de coefficient effectif $\mathrm{D}^{*}$ et le coefficient de dispersion mécanique $\mathrm{D}_{\mathrm{m}}$ (Freeze et Cherry, 1979).

$$
\mathrm{D}=\mathrm{D}^{*}+\mathrm{D}_{\mathrm{m}}
$$

où $\mathrm{D}_{\text {}}$ est une fonction de la vitesse d'écoulement; si v $\rightarrow 0, \mathrm{D}_{\mathrm{m}} \rightarrow 0$ et l'équation (2) se réduit à la seconde loi de Fick deccrivant le transport transitif et diffusif à travers un milieu poreux. Rowe (1987) a démontré que la dispersion mécanique était relativement insignifiante par rapport à la diffusion pour les cas typiques des barrières argileuses quand la vitesse d'écoulement (loi de Darcy) est $\leq 0,1 \mathrm{~m} / \mathrm{an}$. La solution analytique de l'équation (2) a été présentée pour différentes conditions initiales, par Van Genutchen et Parker (1984), et a été adaptée au cas du transport des contaminants à travers une barrière argileuse saturée par Schackelford (1990) sous forme d'abaques.

\section{2}

\section{Cas des sols non saturés}

Les sols utilisés pour construire une barrière d'étanchéisation sont souvent non saturés. Ceci est particulièrement vrai quand la barrière est considérée comme étant une portion de sol naturel ou une barrière d'argile compactée séparant les déchets et la nappe d'eau. Dans ce cas les mesures de $D^{*}$ pour des sols non saturés représentent l'approche la plus réaliste du problème liế au système d'imperméabilisation.

Les écoulements dans les sols non saturés font intervenir des phénomènes physiques complexes liés à la nature polyphasique du milieu poreux qu'est le sol. L'eau contenue dans le sol est caractérisée, d'une part, par le volume qu'elle occupe dans les vides du sol et, d'autre part, par son état énergétique. Cette eau peut circuler librement dans les pores de grandes dimensions ou être retenue dans les pores les plus fins.

Les équations utilisées pour déterminer $\mathrm{D}^{*}$ telles que celles présentées par Jost (1960), Crank (1975) et Muurinene (1990) sont applicables pour les essais sur les sols non-saturés si la teneur en eau volumétrique, $\theta$, est utilisée à la place de la porosité n (Schackelford, 1991). La teneur en eau volumétrique est définie comme suit:

$$
\theta=n S_{r}
$$

où $S$, est le degré de saturation.

Cette teneur peut être déterminée par exemple, à partir de la courbe de caractéristiques hydriques d'un sol donné (Fig. 3). On remarque, en général, que la succion augmente lorsque la teneur en eau diminue. Le compactage des sols comme dans le cas des barrières d'étanchéisation a aussi une influence sur les caractéristiques hydriques, car les dimensions des pores sont complètement modifiées.

Il est important de signaler que, lors des essais de diffusion sur les sols non saturés, le contrôle de la succion des sols est requis afin d'empêcher l'écoulement des contaminations dû à des gradients de succion. Il est enfin reconnu, bien que des expériences sur des sols

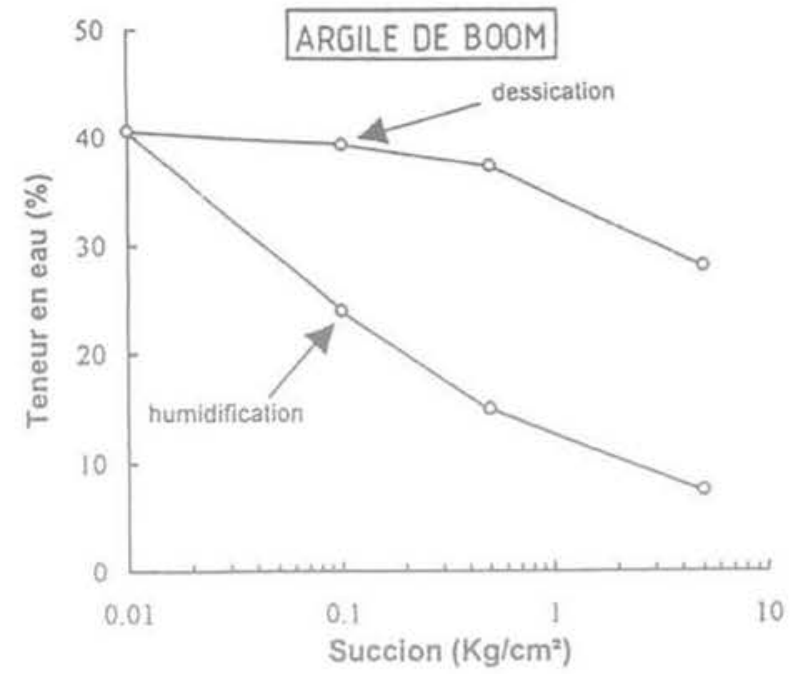

FG. 3 Courbes de caractéristiques hydriques de I'argile de Boom (Belgique) - Van Impe (1974).

Soil-water characteristic curve of Boom clay Van Impe (1974).

non saturés aient déjà été présentées (Conca et Wright, 1990), que ce genre d'essais est assez compliqué à élaborer. Mais l'avènement des centrifugeuses ces dernières années a facilité l'étude de la diffusion dans des sols non saturés. La modélisation est abordable, et les résultats sont assez prometteurs (Sills et Mitchell, 1995).

Les coefficients de diffusion dans les sols non saturés sont généralement inférieurs à ceux mesurés dans les sols saturés. Ceci est dû à l'augmentation de la tortuosité des chemins d'écoulement dans les sols non saturés. Par conséquent, si on utilise des données associées à l'état de saturation du sol, le calcul sera très conservatif. Schackelford (1991) a montré que les valeurs des coefficients de diffusion pour des solutions non-réactives et réactives dans des sols saturés sont 10 à 20 fois plus élevées que celles obtenues dans des sols non saturés (tableau II).

En résumé, on peut dire qu'en l'absence d'écoulements couplés, le transport diffusif des contaminants représente le cas limite du processus de transport à travers une barrière argileuse. A notre avis, la valeur minimale de la conductivité hydraulique d'une barrière argileuse représente une condition nécessaire mais pas suffisante. Le phénomène de diffusion doit être pris en compte lors de l'élaboration et la construction d'un site de décharge. Il est aussi important de considérer dans le futur le cas de sols non saturés afin d'éviter un calcul pénalisant et trop conservatif.

TABLEAUII Variation de coefficient de diffusion D* par $10^{-10} \mathrm{~m}^{2} / \mathrm{s}$ (d'après Schackelford, 1991).

\begin{tabular}{c|c|c}
\hline Type dé solutions & Sols saturés & Sols non saturés \\
\hline non réactives & $1-18$ & $0,63-15$ \\
réactives & $0,54-25$ & $0,04-1,55$ \\
\hline
\end{tabular}




\section{Prescriptions réglementaires pour la construction des barrières d'étanchéisation}

Le type du système d'étanchéité des sites de décharges varie d'un pays à l'autre; il est parfois différent au sein du même pays (cas de la Belgique par exemple). Cette variation dépend de plusieurs facteurs, les stratégies et les pratiques de la gestion des déchets au niveau des autorités locales, la pression des mouvements écologiques, la volonté politique, des raisons principales dictant le choix d'un système d'étanchéité donné. Cependant, toutes les agences de protection de l'environnement ou organisations gouvernementales s'accordent sur un point, c'est la nécessité d'avoir une décharge protégeant l'environnement. Compte tenu de ce consensus, la plupart des décharges modernes sont construites suivant le principe que le dispositif d'imperméabilisation soit capable, primo: d'éliminer entièrement toute fuite de liquide (jus de décharge) et plus particulièrement de prévenir tout risque de fuite dans le sous-sol; secundo: de maintenir en toute sécurité et à long terme un état d'étanchéité parfaite.

Ces exigences sont plus ou moins satisfaites grâce à l'utilisation de système de barrières mixtes où un géotextile et/ou une géomembrane est utilisé(e) conjointement avec une barrière argileuse pour former un dispositif d'étanchéité imperméable. Il est aussi important de souligner que la combinaison des géomembranes et des géotextiles varie considérablement en fonction des possibilités d'application et des différents systèmes en vigueur localement.

\section{1}

\section{Conception des barrières d'imperméabilisation}

La nature des sites de décharges est dictée par le type de déchets à stocker. Les déchets peuvent être classés d'une manière générale en trois catégories:

Type 1 : inertes;

Type 2: ménagers et industriels non dangereux;

Type 3 : industriels dangereux et toxiques.

La classification des décharges correspond généralement à ces catégories de déchets. Dans ce texte on désigne par décharges du type 1 celles qui reçoivent les déchets inertes, ménagers et industriels non dangereux. Une brève présentation sera aussi faite pour la catégorie des déchets dangereux et dans ce cas on fera référence à des décharges de type 2 .

La classification donnée ci-dessus varie considérablement d'un pays à un autre. La figure 4 indique par exemple une variation régionale très notable dans les prescriptions belges. Notons que pour la région de Bruxelles, les déchets sont incinérés ou exportés vers les régions limitrophes (Vam Impe et Bouazza, 1995a). En Allemagne, une distinction est faite entre le Sud et le Nord du pays. En France, les sites de décharges sont répartis en trois “classes», respectivement dénommées III pour les déchets inertes, II pour déchets ménagers et/ou industriels non dangereux et I pour les déchets dangereux (Come, 1994). Comme indiqué, pour chaque

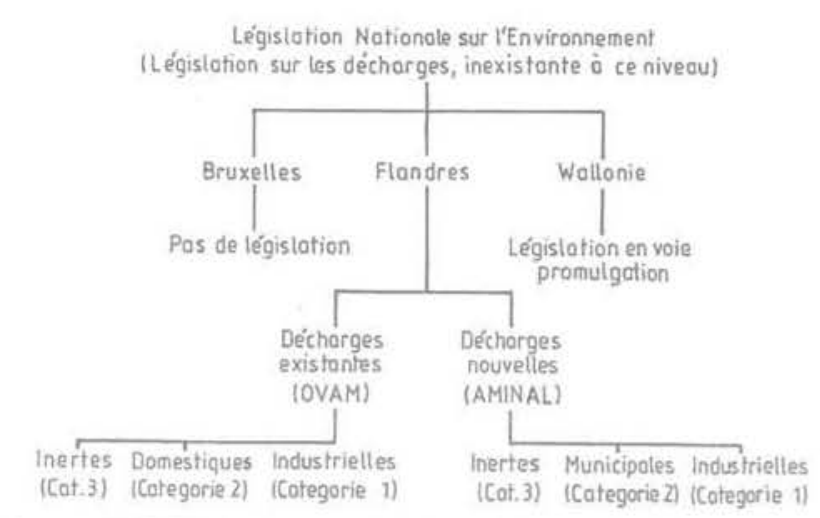

FG. A Système de classification des déchets et des décharges en Belgique,

Belgian classification system for wastes and landfills.

type de décharges, les prescriptions réglementaires changent remarquablement à travers 1'Europe.

\section{A.4.1 \\ Décharges de type 1}

Le but d'un dispositif d'étanchéité est de diminuer le plus possible la migration des contaminants. Pour que cet objectif soit atteint d'une manière efficace, on a recours à un dispositif où il $\mathrm{y}$ a une succession de matériaux permettant d'assurer le drainage et l'étanchéité des décharges. Depuis plusieurs décennies, le seul moyen pratique employé pour parer à une éventuelle pollution du sol était l'utilisation d'une barrière argileuse (Fig. 1). Une autre alternative a vu le jour au début des années 80 par l'utilisation des géomembranes comme barrière d'étanchéisation. Dans ce cas, la membrane repose directement sur le substratum naturel sans qu'il y ait une barrière d'argile compactée. Celle-ci est recouverte par une couche drainante contenant le système de collecte et d'évacuation des lixiviats. Enfin, une couche filtrante est placée entre les déchets solides et le drainage (Fig. 5).

Ce concept insiste beaucoup plus sur l'atténuation de la migration de la pollution que sur sa rétention ou confinement. Cependant, l'émergence de la technologie géosynthétique dans les années 80 et le remarquable progrès technique fait dans ce domaine on fait que le concept de rétention est devenu beaucoup plus réaliste et réalisable. La plupart des décharges modernes sont maintenant construites avec une barrière composite oủ la géomembrane est placée directement sur l'argile compactée.

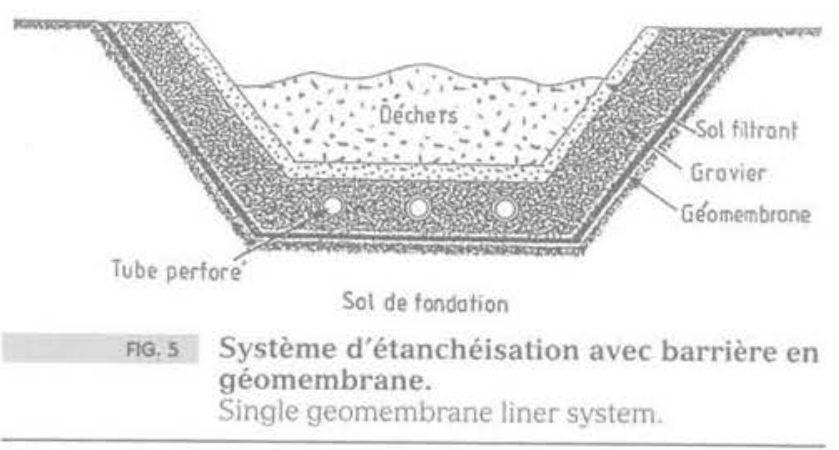



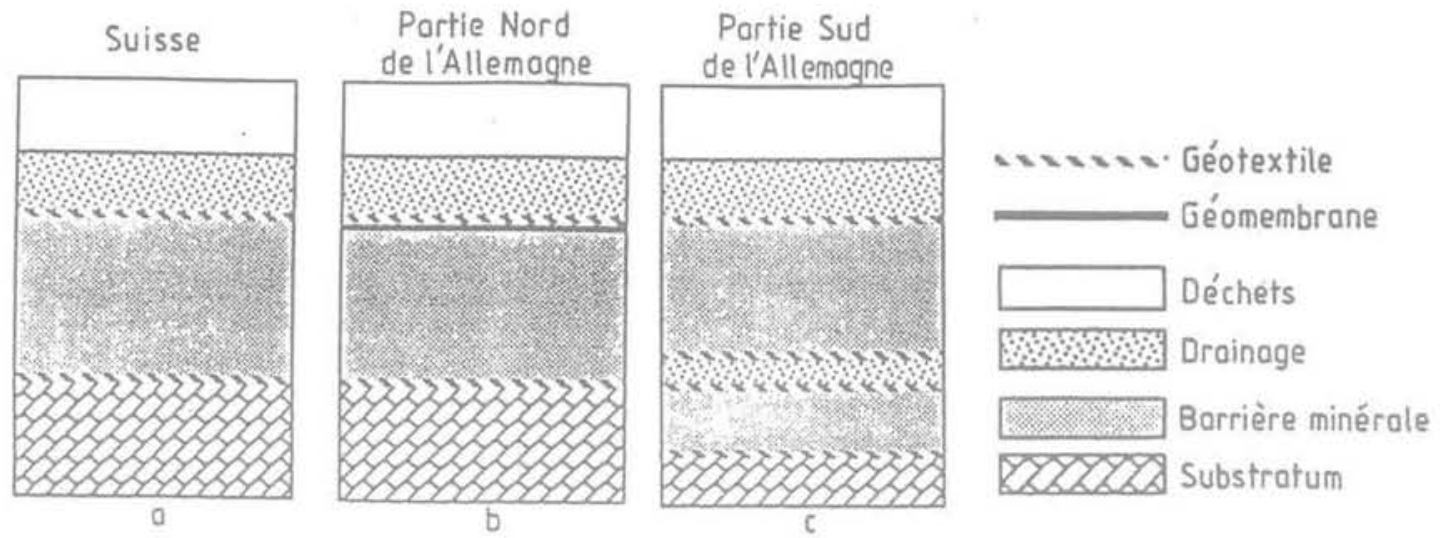

FG.6 Système de construction des barrières d'étanchéisation en Suisse et en Allemagne (d'après Amman and Martinenghi, 1993).

Liner system construction in Switzerland and in Germany (after Amman and Martinenghi, 1993).

Le taux de fuite à travers une barrière mixte parfaite est inférieur à celui qu'on a à travers une membrane ou une argile compactée. En effet, dans une argile compactée sans protection, l'écoulement se fait sur la totalité de la surface de la barrière à cause de l'inévitable microfissuration. En outre, il est très difficile d'installer une membrane sur un site donné et sur une très grande surface sans qu'il y ait des défectuosités. Dans ce cas, le liquide pourra s'écouler librement à travers ces défectuosités. Avec une barrière mixte, le liquide pourra toujours s'écouler librement à travers la membrane. Mais il sera empêché de poursuivre son chemin par une barrière très peu perméable. Par conséquent, si on place un sol peu perméable (argile ou argile silteuse), la fuite à travers une membrane sera minimisée ou réduite à sa plus simple expression. Les analyses théoriques et les essais faits par Giroud et Bonaparte (1989) ont montré que le taux de fuite à travers une barrière mixte, dû à l'existence d'une perforation dans la membrane, est inférieur de plusieurs ordres de grandeur au taux de fuite à travers la même perforation dans une membrane donnée.

Comme il a été spécifié précédemment, la variation des prescriptions réglementaires concernant les dispositifs d'étanchéité d'un pays à un autre est très courante. La figure 6 donne un exemple de barrière d'étanchéité utilisée en Suisse et en Allemagne. On peut remarquer des différences dans leur conception. La raison pour laquelle la conception Suisse n'inclut qu'une couche drainante et des géotextiles tient au fait que tous les déchets ménagers sont incinérés et stockés sous forme de cendres volantes (Amman et Martinenghi, 1993). Le Sud de I'Allemagne utilise un concept similaire. Cependant, dans les deux cas la résistance optimale à la migration des contaminants n'est pas prévue, du fait de l'absence de membrane couvrant la couche d'argile. Dans le Nord de l'Allemagne, le but du géotextile utilisé est différent. Le géotextile primaire couvant la membrane est un géotextile épais ayant une résistance élevée au poinçonnement, permettant ainsi la protection de la membrane contre d'éventuelles perforations ou des dégâts semblables, causés par des objets tranchants non spécifiés dans la décharge. Le second géotextile joue un rôle de renforcement de la barrière argileuse. Il est important de souligner que l'existence de deux solutions distinctes en Allemagne est due à leurs structures géologiques. Dans le Nord, le

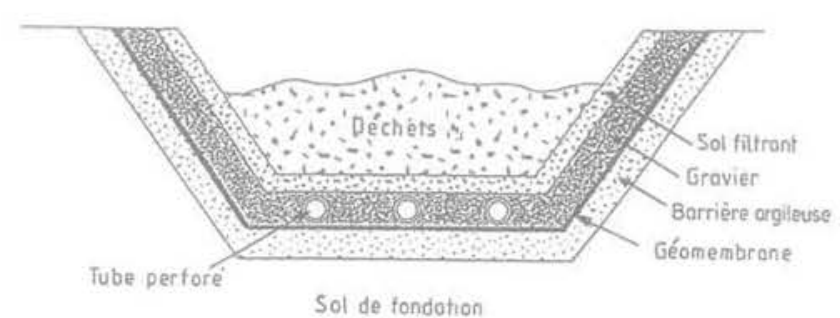

FIG.7 Barrière d'étanchéisation minimale aux États-Unis.

Minimum liner system in the United States.

bedrock se trouve à une profondeur de 60 à $70 \mathrm{~m}$, alors que dans le Sud il est beaucoup plus profond. Aux Etats-Unis, la conception minimale consiste en un système d'étanchéité composite simple (géomembrane) argile compactée) avec un système de collecte et d'évacuation des lixiviats (Fig. 7). Dans ce cas, la couche drainante a une conductivité hydraulique $\geq 1 \mathrm{~cm} / \mathrm{s}$, et l'épaisseur minimale de la membrane doit être $\geq 0,76 \mathrm{~mm}$.

Le GLR européen (ETC 8, 1993) recommande aussi la même conception, mais avec une légère différence

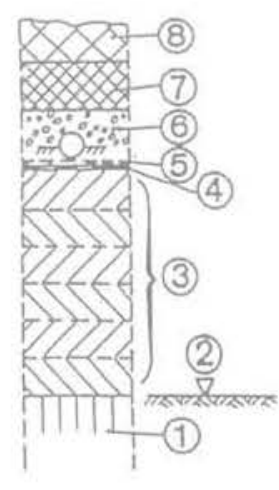

\begin{tabular}{|c|l|}
\hline No & \multicolumn{1}{|c|}{ Couche } \\
\hline 1 & Substratum \\
2 & Couche de base \\
3 & Couche argileuse \\
& première couche \\
& deuxième couche \\
& troisième couche \\
& dernière couche pour chaque cos \\
4 & Géomembrone \\
5 & Couche protectrice \\
6 & Topis drainant \\
7 & Couche de transition (si nécessoire) \\
8 & Déchets \\
\hline
\end{tabular}

FG. 8 Système de barrière d'étanchéisation composite proposé par ETC 8. Composite basal lining system proposed by ETC 8. 


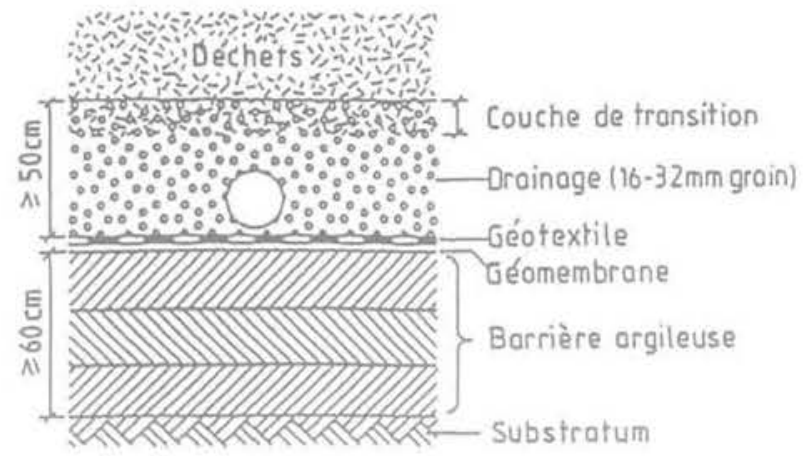

FG.9 Barrière d'étanchéisation composite standard en Autriche (d'après Brandl, 1992).

Standard scheme of composite base sealing in Austria (after Brandl, 1992).

concernant l'épaisseur minimale de la barrière argileuse et la protection de la membrane (Fig. 8). Le système d'étanchéité composite simple (Fig. 9) est aussi en vigeur en Autriche (Brandl, 1992) avec une couche drainante $\geq 0,5 \mathrm{~m}$. Come (1994) a présenté un système d'étanchéité (Fig. 10) où la membrane est séparée de la couche d'argile par une couche de pose. On peut remarquer que dans toutes les conceptions présentées précédemment la membrane repose directement sur la barrière argileuse. Ce choix est fait de manière à ce qu'il y ait un bon contact hydraulique (contact intime). Généralement, on ne sépare pas la membrane et la barrière argileuse par un géotextile ou une couche de matériaux très perméable. Autrement le contact hydraulique sera influencé, et la résistance à la migration de la pollution sera faible. Le concept de «contact intime », dans les systèmes d'étanchéités composites, dans les sites de décharges, n'est pas nouveau.

L'approche du concept de (contact intime ) est faite dans l'intention de minimiser la migration ou l'écoulement latéral du liquide qui pourrait passer à travers une

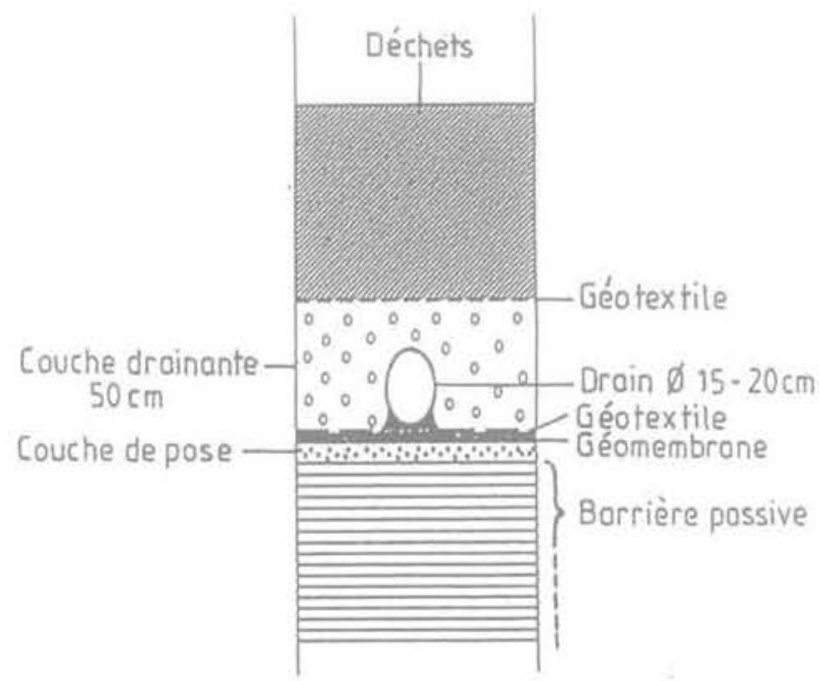

FIG.10 Barrière d'étanchéisation composite (simple) utilisée en France (d'après Come. 1994).

(Single) composite liner used in France (after Come, 1994). perforation de la membrane. Ce concept augmente ou encourage la contribution de la partie sol constituant le système composite en minimisant les fuites à travers la barrière d'étanchéité et déconseille l'utilisation d'un géotextile ou d'un matériau très perméable sous la membrane. Des études récentes (Estornell et Daniel, 1992) ont montré que la présence directement sous la membrane d'un milieu drainant ayant une transmissivité élevée (géotextile ou sable) peut augmenter le taux de fuite à travers le dispositif d'étanchéité comparé à un système composite ayant un bon contact entre ses composants. On peut remarquer aussi que dans la réglementation américaine la protection de la membrane n'est pas requise, tandis que dans les recommandations du GLR européen, présentement en discussion au sein du CT5 (SIMSTF), la protection est bien requise.

\section{9 \\ Décharge de type 2}

Les déchets dangereux et toxiques représentent un très haut risque pour l'environnement et la santé publique. Deux événements reliés à la pollution industrielle ont alerté l'opinion publique du danger que représente ces types de déchets et des conséquences qui peuvent en découler. Il s'agit de la pollution par le mercure le long de la baie de Minamata au Japon (fin des années 60) et la contamination de site du canal de Love au États-Unis (début des années 80). En Russie, le délabrement des gigantesques concentrations d'industries lourdes, chimiques, nucléaires et d'armements engendre une pollution phénoménale. Près de 2 millions de tonnes de déchets toxiques sont simplement stockés (sur un total de plus de 80 millions de tonnes de déchets en tous genres) (Anon, 1995). Dans les pays en voie de développement la situation est plus grave encore.

La nature des déchets de type 2 joue un rôle important dans la conception du dispositif d'étanchéité. Cette conception est différente de celle en vigueur pour les décharges de type 1 et varie aussi d'un pays à un autre.

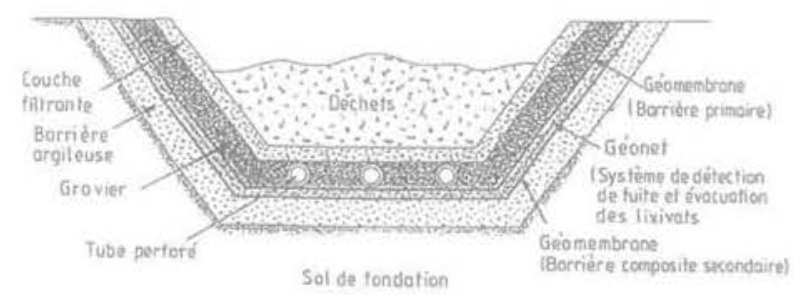

FG.11 Barrière d'étanchéisation composite (double). Double liner system.

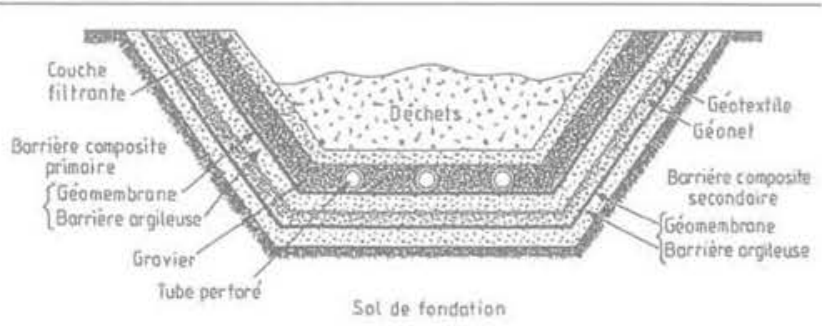

คตG. 12 Barrière d'étanchéisation composite (double).

Double liner system. 
Aux États-Unis, l'utilisation du système d'étanchéité composite multiple est systématique (Fig. 11 et 12). Généralement, il est constitué d'une zone filtrante qui sépare les déchets et la zone de drainage libre ou système de collecte et d'évacuation primaire (SCELP). Dans un système parfait, le lixiviat est arrêté par la membrane et évacué pour traitement. Cependant, des études récentes ont montré que des fuites à travers le SCELP peuvent se manifester (Bonaparte et Goss, 1990). Un système de détection de fuite ou système de collecte et d'évacuation secondaire des lixiviats (SCELS) est généralement placé sous la barrière primaire. Il a la même fonction que le SCELP. Sous le SCELS, il y a la barrière secondaire qui est une barrière composite simple constituée d'une membrane et d'une couche d'argile compactée. Le SCELP et le SCELS ont une épaisseur minimale de $30 \mathrm{~cm}$. Une autre alternative est illustrée par la figure 12. Le système primaire d'évacuation des lixiviats (SCELP) et le système secondaire (SCEL,S) sont similaires à ceux décrits dans la figure 11. Cependant, les barrières primaire et secondaire sont des barrières composites simples. L'approche américaine est différente de l'approche européenne telle que présentée par le GLR (ETC8, 1993). En effet, la conception européenne insiste beaucoup sur l'utilisation de matériaux naturels et minimise l'utilisation de la géomembrane (Fig. 8). La seule différence avec la conception pour les décharges de type 1 est l'épaisseur minimale de la barrière argileuse qui doit être $\geq 3 \mathrm{~m}$.

Bien que les géomembranes aient une conductivité hydraulique très faible (de l'ordre de $3.10^{-15} \mathrm{~m} / \mathrm{s}$ à $3.10^{-13} \mathrm{~m} / \mathrm{s}$ avec $\mathrm{H}_{2} \mathrm{O}$ comme perméant) et qu'elles soient hautement résistantes à la dégradation biologique du fait de leurs poids moléculaires (Kœrner et al., 1992), elles ne sont pas totalement résistantes, d'une part, à l'écoulement de l'eau et aux produits chimiques et, d'autre part, les liquides organiques peuvent causer le gonflement du polymère et changer ainsi ses propriétés. Rowe (1991) a attiré l'attention sur le fait que les géomembranes peuvent jouer un rôle efficace de barrière pendant approximativement 25 ans mais leur aptitude à contenir les lixiviats au-delà de cette durée est mal connue. Giroud et Cazuffi (1989) ont montré. par exemple, que la perméabilité du polyéthylène était beaucoup plus élevée avec du benzène ou du xylène qu'avec de l'eau. Ils ont reconnu qu'il y a encore beaucoup de choses à apprendre sur la résistance chimique et la durabilité des géomembranes utilisées pour la protection de l'environnement. En résumé, la géomembrane ne doit pas être considérée comme la panacée dans la construction des dispositifs d'étanchéité. Les réticences à son utilisation sont principalement dues au manque de données sur son comportement à long terme, dans un milieu agressif. Cependant, à notre avis, dans des situations critiques telles que le stockage des déchets dangereux, l'utilisation d'un système d'étanchéité composite multiple avec les protections adéquates représente une sécurité supplémentaire.

\section{2}

\section{Utilisation des géocomposites ou matelas bentonitique}

La barrière argileuse compactée est clairement le matériau traditionnel de base requis dans toutes les prescriptions réglementaires. Une étude récente faite

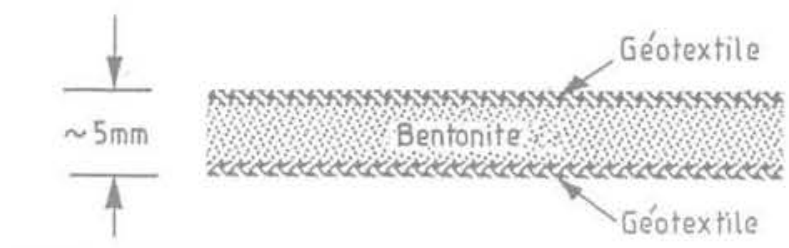

FG 13 Exemple de géocomposite courant. Example of geosynthetic clay liner (GCL).

dans les 50 États constituant les États-Unis sur les dispositifs d'étanchéité utilisés dans les décharges de type 1 (Fahim et Kœrner, 1993) a montré:

- qu'une barrière argileuse simple placée directement sous les déchets est utilisée dans 19 États;

- qu'une barrière argileuse faisant partie d'un système composite simple est utilisée dans 20 États.

Cependant, depuis un certain temps on assiste à l'introduction de géocomposites comme remplacement possible des barrières argileuses dans la conception des dispositifs d'étanchéité. Notamment sur les flancs des décharges ou dans un dispositif d'étanchéité composite multiple oú il serait difficile de compacter sans détériorer la géomembrane.

Le géocomposite est constitué d'une couche mince d'argile bentonitique maintenue en sandwich entre deux couches de géotextile (Fig. 13). La bentonite est connue pour son potentiel de gonflement élevé et sa capacité absorbante. Quand elle est humidifiée, la bentonite devient pratiquement imperméable. Parmi les types de bentonite existants, la bentonite sodique prédomine dans les géocomposites produits en Amérique du Nord, alors que la bentonite calcique est généralement utilisée dans les géocomposites produits en Europe. Madsen et Nuesch (1994) donnent plus de détails dans ce domaine.

La maîtrise de toutes les facettes d'un matériau innovant est toujours compliquée.

\section{4}

\section{Étude de cas}

L'étude a été faite par le Laboratoire de Mécanique des sols-RUG à Gand, sur un site où des réservoirs décoratifs ont été construits avec un dispositif d'étanchéité comprenant un géocomposite. La surface de ces types de réservoirs varie entre $150 \mathrm{~m}^{2}$ à $800 \mathrm{~m}^{2}$. Après que le géocomposite ait été placé, il a été recouvert avec du béton " $\mathrm{sec}$ ) (150 kg ciment $/ \mathrm{m}^{3}$ et gravier $\left.4 / 20\right)$ mélangé à une très faible teneur en eau. Le problème a commençé à se poser après que le réservoir ait été rempli d'eau. Les mesures in situ indiquaient que l'eau s'infiltrait à travers la barrière d'étanchéité à un certain débit non négligeable. Le maitre d'œuvre pensait à une possible réaction chimique entre la bentonite et le ciment utilisé pour préparer le béton sec. Dans ce cadre, le Laboratoire de Mécanique de l'Université de Gand a été sollicité pour conduire toute une série d'essais dans les conditions du site.

Une première série d'essais de conductivité hydraulique a été effectuée sur le géocomposite «non hydraté $(\mathrm{sec})$ n afin d'établir une référence de base (Fig. 14). Une conductivité hydraulique (k) de $4.10^{-11} \mathrm{~m} / \mathrm{s}$ a été obtenue après 11 jours de perméation et s'est stabilisée à ce 


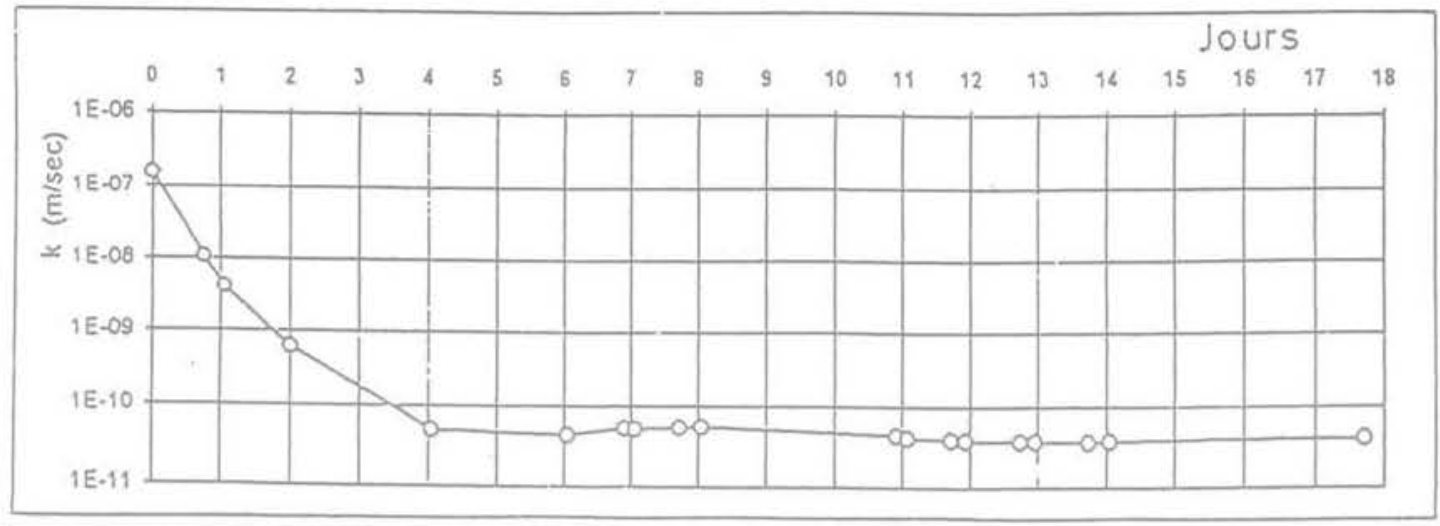

Universiteit - Gent

Laboratorium voor Grondmechanica

FG. 14 Résultat de Yessai de perméabilité sur le géocomposite de référence.

Permeability test results on GCL (dry) for reference : case of a site in Ghent-Kennedy Bouleyard.

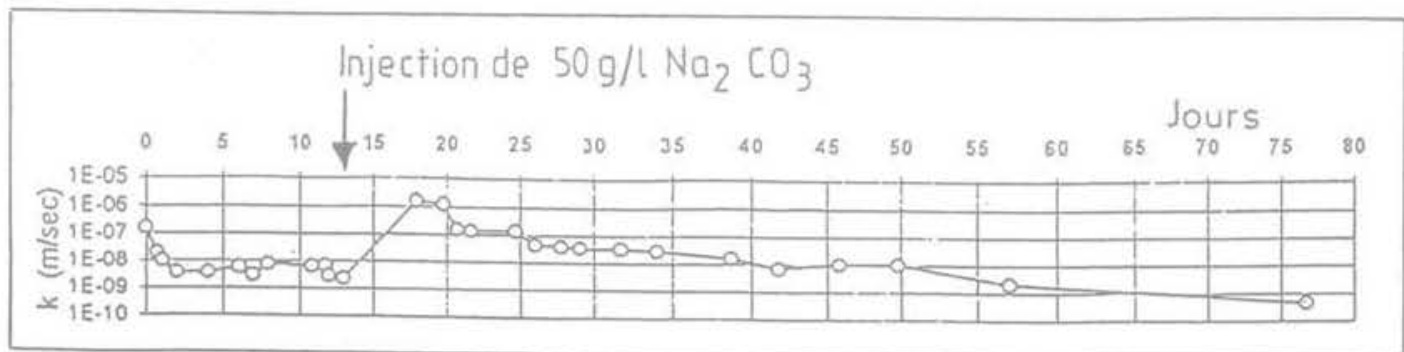

Universiteit - Gent

Laboratorium voor Grondmechanica

FIG. 15 Résultat de l'essai de perméabilité sur le géocomposite + béton sec.

Permeability test results on GCL + dry concrete : case of a site in Ghent-Kennedy Boulevard.

niveau. Il faut souligner que les valeurs obtenues entre 0 et 3 jours correspondent au temps nécessaire à la bentonite pour s'humidifier. En réalité, elles représentent la quantité d'eau que la bentonite a absorbé pour être dans un état humide. Après ce stade, l'épaisseur du géocomposite est passée de $5,4 \mathrm{~mm}$ à $10,3 \mathrm{~mm}$.
La seconde série d'essais concernait l'échantillon de géocomposite (sec) surmonté du mélange de béton «sec » (Fig. 15). On peut remarquer que la conductivité hydraulique s'est stabilisée aux environs de $2.10^{-9} \mathrm{~m} / \mathrm{s}$ après 13 jours d'essais de perméation. La raison pour laquelle la valeur de ( $\mathrm{k}$ ) a augmenté brusquement peu

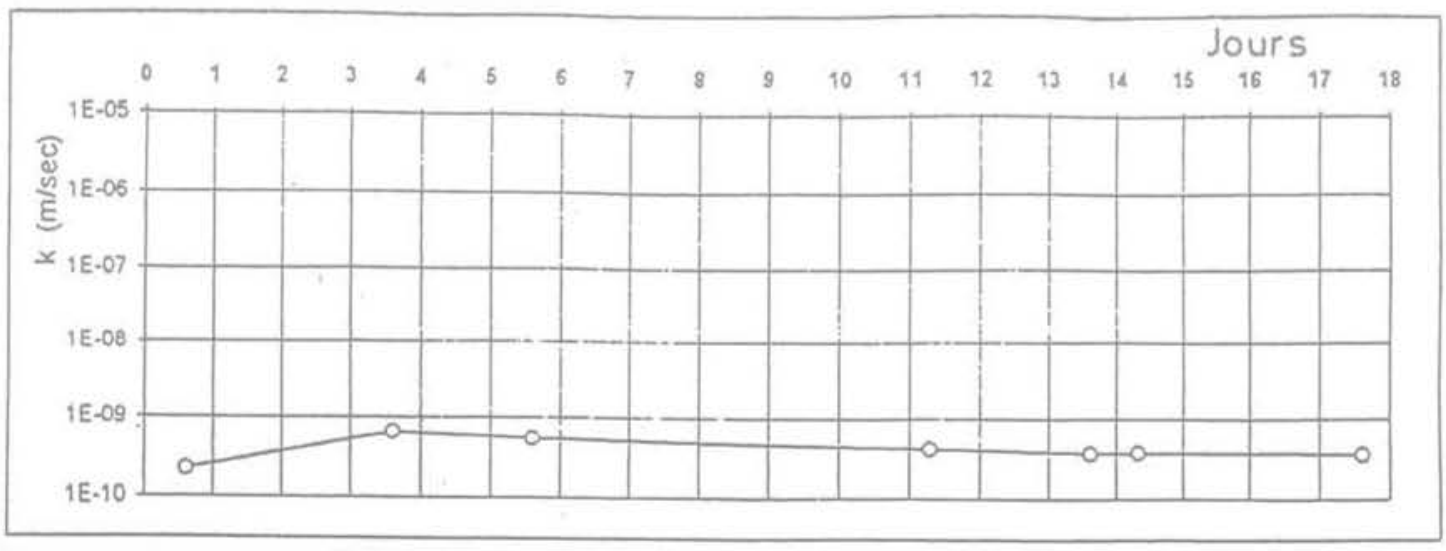

Universiteit - Gent

Laboratorium voor Grondmechanica

Fig. 16 Résultat de l'essai de perméabilité sur le géocomposite provenant du site de Gand-Kennedy boulevard. Permeability test results on GCL from the site of Ghent-Kennedy Boulevard. 
après est due au fait que l'essai a été stoppé à 13jours pour injecter une solution de $\mathrm{Na}_{2} \mathrm{CO}_{3}(50 \mathrm{~g} / \mathrm{l})$ dans le géocomposite, et aussi au durcissement du béton; l'essai a repris normalement au $18^{e}$ jour, la valeur de $(\mathrm{k})$ obtenue après 75 jours était de $8.10^{-10} \mathrm{~m} / \mathrm{s}$.

La troisième et dernière série concerne l'échantillon du géocomposite provenant du site, La figure 16 montre que le géocomposite est déjà hydraté à cause du passage de l'eau; la valeur moyenne de $(\mathrm{k})$ obtenue est de $5.10^{-10} \mathrm{~m} / \mathrm{s}$. Les valeurs de $(\mathrm{k})$ mesurées dans les trois cas sont toutes acceptables. Les seules raisons qui pouvaient avoir causées la fuite constatée sur site sont:

1) l'eau du réservoir contenait des polluants ou

2) le placement du géocomposite a été très mal fait.

Bien que le géocomposite ait été placé sans préhumidification préalable (ce qui n'est pas conseillé), la possibilité (1) est à rejeter car l'eau n'était pas polluée à un haut degré; il n'y avait pas d'interaction à attendre avec la bentonite. La possibilité (2) semble la plus plausible; Daniel (1993) a d'ailleurs attiré l'attention sur le fait de maîtrise sur site d'un tel type de matériau.

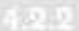

\section{Épaisseur minimale et déformabilité de la barrière argileuse}

Les prescriptions réglementaires en vigueur dans plusieurs pays spécifient une épaisseur minimale de la barrière argileuse. Cependant, aucun consensus n'a été atteint sur une valeur minimale standard. En GrandeBretagne, une barrière de $1 \mathrm{~m}$ d'épaisseur est généralement requise. En Belqique, une épaisseur minimale $\geq 1 \mathrm{~m}$ est une pratique courante. En Suisse, l'épaisseur minimale est de $0,8 \mathrm{~m}$. Les recommandations du GLF (ETC 8, 1993) proposent une épaisseur minimale $\geq 0,75 \mathrm{~m}$ pour les décharges de type 1 et $\geq 3 \mathrm{~m}$ pour les décharges de type 2. En Allemagne l'épaisseur minimale pour les décharges de type 2 est de $1,5 \mathrm{~m}$. Tandis que pour les décharges du type 1, elle est similaire à celle proposée par le GLR européen. Aux États-Unis, I'EPA requiert une épaisseur minimale de $0,6 \mathrm{~m}$ pour les décharges du type 1 et $0,9 \mathrm{~m}$ pour les décharges du type 2. Cependant les différents Etats sont libres d'imposer une épaisseur minimale différente. Gordon et al. (1990) ont cité le cas de l'état de Wisconsin où l'épaisseur minimale requise est de $1,5 \mathrm{~m}$. Les travaux récents présentés par Benson et Daniel (1994a, 1994b) ont montré que les barrières arqileuses, dont l'épaisseur est inférieure à $0,3 \mathrm{~m}$, ont tendance à ètre 10 à 100 fois plus perméables que les barrières dont l'épaisseur est au moins égale à $0,6 \mathrm{~m}$. Pour les épaisseurs entre $0,6 \mathrm{~m}$ et $0,9 \mathrm{~m}$, la grandeur et la dispersion de la conductivité hydraulique étaient insensibles à l'épaisseur. Ils ont aussi conclu qu'une barrière plus épaisse peut être recommandée pour tenir compte d'un nombre de facteurs spécifiques à un site donné (ex. augmenter la capacité d'atténuation géochimique d'une barrière)

Outre le fait qu'une barrière argileuse soit construite de manière à empêcher la migration des lixiviats, elle est aussi sujette à des déformations causées par des tassements différentiels. Quelle déformation doit-on tolérer sans qu'il y ait une augmentation inadmissible de la perméabilité de la barrière argileuse? Scherbeck (1992) s'est intéressé à ce problème, il a conclu que si les déformations causent un mécanisme de cisaillement local, les changements dans la conductivité hydraulique seront faibles. En outre, la formation de fissures mènera à la ruine de la barrière argileuse. Scherbeck (1992) a développé une approche afin de vérifier si la déformation entraînera une fissuration stable.

Il est néanmoins de notre avis qu'il n'est pas recommandé de consacrer, dans le futur, des activités excessives de recherche dans ce domaine, afin de développer des essais ou d'essayer d'éviter ce processus mécanique de fissuration de la barrière argileuse. Il est en tout cas pratiquement impossible de bannir la microfissuration due au tassement différentiel. En revanche, l'effort de recherche devrait être concentré dans le développement de techniques d'essais in situ afin d'évaluer la diffusion et mesurer la conductivité hydraulique incluant dans l'essai l'inévitable effet de la fissuration de la barrière argileuse.

Le problème de dessication des barrières, causant retrait, fissuration et augmentation de la conductivité hydraulique a été abondamment discuté par Mitchell (1991), Doll et Wessolek (1994)... Récemment encore, Jessberger (1995) a conclu que du point de vue géotechnique plusieurs de ces problèmes restent sans solution, bien qu'à partir de a l'expérience pratique $»$ on admet souvent qu'une barrière acceptable peut être conçue facilement en suivant les prescriptions réglementaires. A notre avis, ce n'est pas toujours aussi simple, et on a encore besoin d'approfondir beaucoup la recherche fondamentale dans ce domaine.

\section{5}

\section{Contrôle de qualité du compactage}

Le compactage de la barrière argileuse est une partie essentielle de la procédure de construction. Il permet d'avoir une fondation dont la résistance sera prolongée et aussi d'éviter d'éventuelles détériorations de la décharge. Ces détériorations peuvent être causées par de larges tassements ou par des tassements différentiels. Il n'est pas suffisant de réaliser une portance adéquate, mais il est primordial d'avoir une portance uniforme de façon à éviter de possibles tassements différentiels. On entend par uniformité, des conditions de sol qui permettent un tassement égal sur l'entière surface compactée, les zones de compactage (faibles» et ("dures») doivent être évitées. En pratique, différents types d'essais sont utilisés pour vérifier la qualité du compactage, en d'autres termes la rigidité du sol compacté. Parmi les essais disponibles, quelques uns ont une large applicabilité, alors que d'autres ne sont utilisés que pour des projets spéciaux ou dans le cas de conditions de sols spéciaux. L'applicabilité des essais est une fonction de la mobilisation de l'équipement, des considérations de coùt et de précision. La figure 17 indique par exemple, que des essais rudimentaires tels que le pénétromètre de poche sont peu coûteux, mais ont une faible précision. Par ailleurs, les essais avec une précision assez élevée, tels le pressiomètre de Ménard, l'essai downhole, etc., sont coûteux mais très instructifs. Dans le cas des barrières argileuses pour sites de décharges, les essais destructifs sont à rejeter par principe, du fait du risque de détériorations qu'ils peuvent engendrer. La procédure d'essai comme dans le cas de la méthode de remplacement ou du nucléodensimètre peut aussi mener à de larges différences dans les résultats (Noorany, 1990) dues à un mauvais étalonnage.

Une nouvelle méthode de contrôle du compactage a été développée au Laboratoire de mécanique de l'Université de Gand (Haegeman et Van Impe, 1995, Haege- 


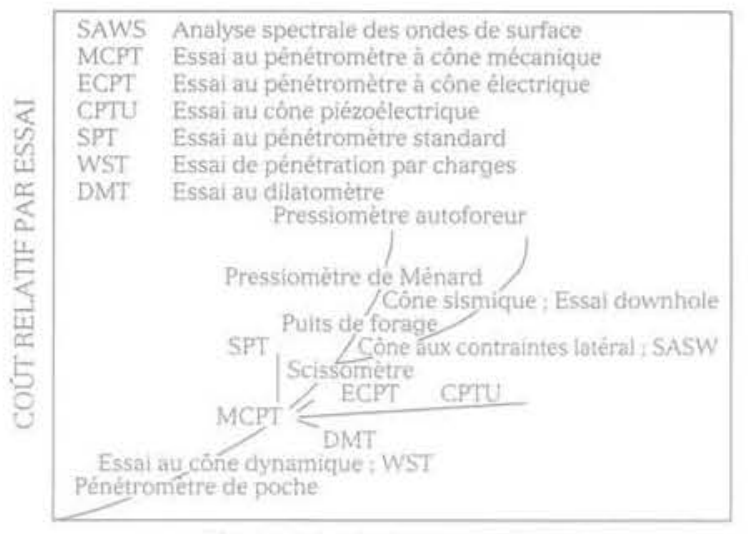

PRECISION RELATIVEDE L.ESSA]

Fic. 17 Relation coût-précision des essais in situ les plus courants.

Cost-accuracy relationship for in situ tests.

man, 1995). C'est un essai non destructif basé sur l'analyse des ondes de surface de Rayleigh. Ce concept n'est pas nouveau, à l'origine il a été développé pour évaluer l'épaisseur et la rigidité des couches de roulement (Van den Poel, 1951).

L'avènement de l'outil informatique et les progrès faits dans l'analyse des signaux et la propagation des ondes ont donné le nom actuel d'analyse spectrale des ondes de surfaces (SASW). Les premiers travaux dans ce domaine ont été présentés par Nazarian et Stockoe (1984), et depuis la méthode a été améliorée pour étudier la liquéfaction des sols, la rigidité des couches de sols à faible déformation, les propriétés dynamiques des sols et des déchets solides (Addo et Robertson, 1992, Mancuso et Vinale, 1993, Haegeman et Van Impe, 1993, Kavazaniian et al., 1994).

\section{1}

\section{Méthode SASW}

Les méthodes utilisant les principes de propagation d'ondes en déterminant les propriétés élastiques sont soient intrusives ou non intrusives. Les méthodes intrusives requièrent un trou de forage ou une pénétration. Elles déterminent directement les vitesses des ondes de compression ( $V_{p}$ ) et de cisaillement ( $V_{\text {) }}$ ). Ces méthodes incluent le cross-hole, le down-hole, le pénétromètre cône sismique, etc. Les méthodes non intrusives déterminent le profil de vitesse des ondes à partir de la surface du sol. Elles incluent la réflexion de surface, la réfraction de surface et l'analyse spectrale des ondes de surface. La réflexion et la réfraction de surface conviennent généralement aux travaux d'exploration géophysiques. Elles ne donnent pas de profils précis du point de vue géotechnique. La méthode SASW utilise la dispersion de l'onde de surface pour déterminer indirectement les vitesses des ondes de cisaillement et de compression. L'avantage de ces mesures, c'est qu'elles soient faites à des niveaux de contraintes et de déformations connus $\left(\leq 10^{-4} \%\right)$ et ne requiérent aucunement le remaniement du sol. La dispersion est exprimée sous forme d'une courbe représentant la variation de la vitesse de propagation avec la longueur d'onde. Une fois la courbe de dispersion in situ déterminée, le profil de rigidité est calculé en utilisant un algorithme d'inversion. L'inversion permet de déterminer un profil d'ondes de vitesse de cisaillement détaillé avec des profils de rigídités simples ou très complexes.

\section{Aspect théorique}

Les ondes de surfaces utilisées dans la méthode SASW sont des ondes de Rayleigh polarisées. Ce sont des ondes sismiques voyageant le long de la surface exposée d'un système solide. Elles ont un mouvement de particules qui diminue avec la profondeur. La profondeur du mouvement de particules est déterminée par la longueur d'onde (ou la fréquence). Les ondes de basse fréquence (ondes longues) s'étendent plus profondément dans le système que les ondes de haute fréquence (ondes courtes). Cette propriété est illustrée par la figure 18.

Les ondes de Rayleigh de longueurs d'ondes courtes se propagent à travers la couche de surface; leurs vitesses seront déterminées par les propriétés de cette couche. En outre; les ondes longues de Rayleigh se propagent à travers les différentes couches existantes, leurs vitesses seront déterminées par les propriétés combinées de ces couches. En résumé, dans un milieu multicouche, la vitesse de propagation de l'onde de surface dépend de la fréquence de l'onde fou longueur d'onde). Cette variation de la vitesse avec la fréquence est apelée "dispersion ».

Toutes les couches du profil peuvent être échantillonées simplement en générant des ondes de surface sur une large palette de longueurs d'ondes ou de fréquences. Les vitesses varieront avec la rigidité et l'épaisseur des couches dans le système. L'objectif de la méthode SASW est de mesurer la dispersion de cette onde de surface.

La vitesse de l'onde de surface $(\mathrm{V})$ d'un matériau est reliée à la vitesse d'onde de cisaillement $\left(V_{5}\right)$. L'onde de surface se propage à une vitesse légèrement inférieure à la vitesse de l'onde de cisaillement. La relation entre la vitesse de l'onde de surface et la vitesse de l'onde de cisaillement dépend du coefficient de Poisson (v) :

$$
\mathrm{V}_{\mathrm{r}} \approx 0,9 \mathrm{~V}_{\mathrm{s}} \text { pour } 0,1 \leq v \leq 0,3
$$

Les valeurs du module de cisaillement et du module d'Young sont données par:

$$
\mathrm{G}=\rho \mathrm{V}_{\mathrm{s}}^{2} \text { et } \mathrm{E}=2 \mathrm{G}(1+v)
$$

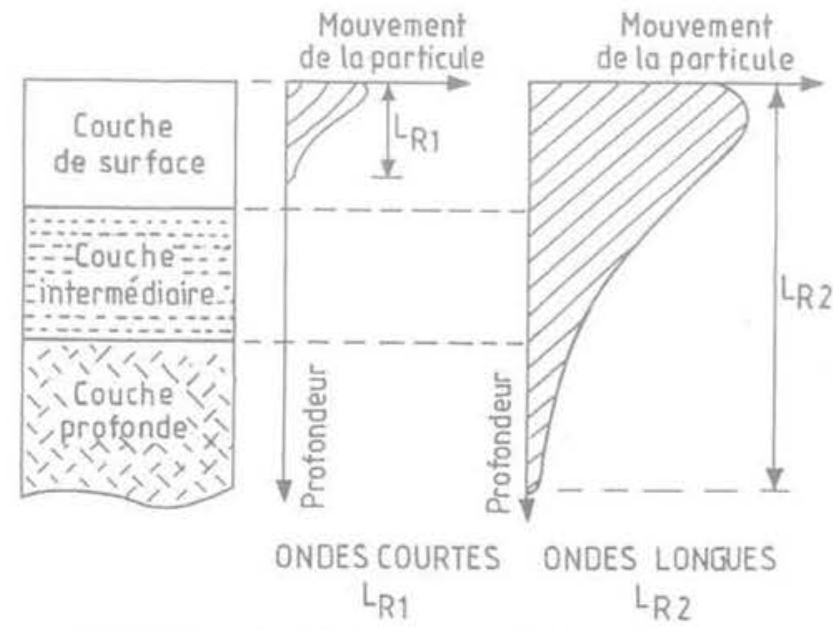

FIG. 18 Distribution en profondeur du mouvement vertical pour deux ondes de surface ayant des longueurs d'onde différentes. Distribution in depth of the vertical motion for two surface waves having different wavelentghs. 


\section{Procédure d'essai}

La configuration générale de la source, capteurs et équipement d'acquisition est montrée sur la figure 19. Les ondes de surface sont générées en appliquant sur la surface du sol un chargement vertical dynamique. La propagation de ces ondes le long de la surface est enregistrée par deux capteurs placés à des distances $\mathrm{d}_{1}$ et $d_{2}$ de la source (marteau). Un analyseur de signaux dynamiques est utilisé afin d'enregistrer et d'analyser les mouvements réceptionnés par les deux capteurs. L'aptitude de calculer rapidement les transformées de Fourier sur site est un avantage essentiel dans la méthode SASW. Elle permet immédiatement à l'opérateur d'avoir une idée sur la qualité des résultats et, si nécessaire de réarranger le placement de la source et des capteurs.

\section{4}

\section{Procédure d'analyse}

Pour chaque espacement source/capteur, le temps enregistré par deux capteurs, $x(t)$ et $y(t)$, est transformé dans le domaine de fréquence résultant du spectre linéaire des deux signaux, $X(f)$ et $Y(f)$. Le spectre combiné des signaux, $G_{x y}$ (f) est alors calculé en multipliant $\mathrm{Y}(\mathrm{f})$ par le conjugué complexe de X(f). En plus du spectre combiné, la fonction de cohérence et le spectre d'énergie de chaque signal sont calculés. Il est à signaler que ces quantités sont calculées en temps réel par l'analyseur de signaux.

Les paramètres importants sont la phase du spectre combiné et la fonction de cohérence. La fonction de cohérence représente le ratio signal/bruit et devrait être égale à 1.

La phase du spectre combiné représente la différence de phase du mouvement enregistrée par les deux capteurs. La vitesse de l'onde de surface (V) et la longueur d'onde ( $\left.L_{1}\right)$ peuvent être déterminées à partir de la phase du spectre combiné $\left(\theta_{x y}(f)\right)$, en utilisant les expressions suivantes:

$$
\left.t(f)=\theta_{x y}(f)\right) / 2 \pi f
$$

où l'angle de phase est en radian et la fréquence, $f$, en Hertz. La vitesse de phase de l'onde de surface est déterminée par:

$$
\mathrm{V}_{\mathrm{r}}(\mathrm{f})=\left(\mathrm{d}_{2}-\mathrm{d}_{1}\right) / \mathrm{t}(\mathrm{f})
$$

et la longueur d'onde correspondante à l'onde de surface est calculée par:

$$
\mathrm{L}_{\mathrm{r}}=\mathrm{Vr} / \mathrm{f}
$$

Le résultat de ces calculs est une courbe de dispersion $\left(V_{r}-L_{r}\right)$ pour chacque espacement de capteur. Ces courbes individuelles sont assemblées en une courbe composite de dispersion spécifique au site.

Pour un système multicouche, la rigidité de sol varie avec la profondeur. Dans ce cas, une procédure d'inversion est requise pour obtenir le profil de rigidité à partir de la courbe de dispersion expérimentale. Cette procédure requiert un artifice de calcul basé sur un profil de vitesse arbitraire correspondant au profil de rigidité. La courbe théorique de dispersion sera ainsi calculée pour ce profil (Nazarian, 1984). Celle-ci est alors comparée avec la courbe expérimentale, et le profil arbitraire sera ajusté afin d'améliorer son calage. Cette procédure est répétée jusqu'à ce que les courbes de dispersion théorique et expérimentale soient complètement étalonnées. Le profil ainsi obtenu représentera le profil de rigidité dans le système étudié.

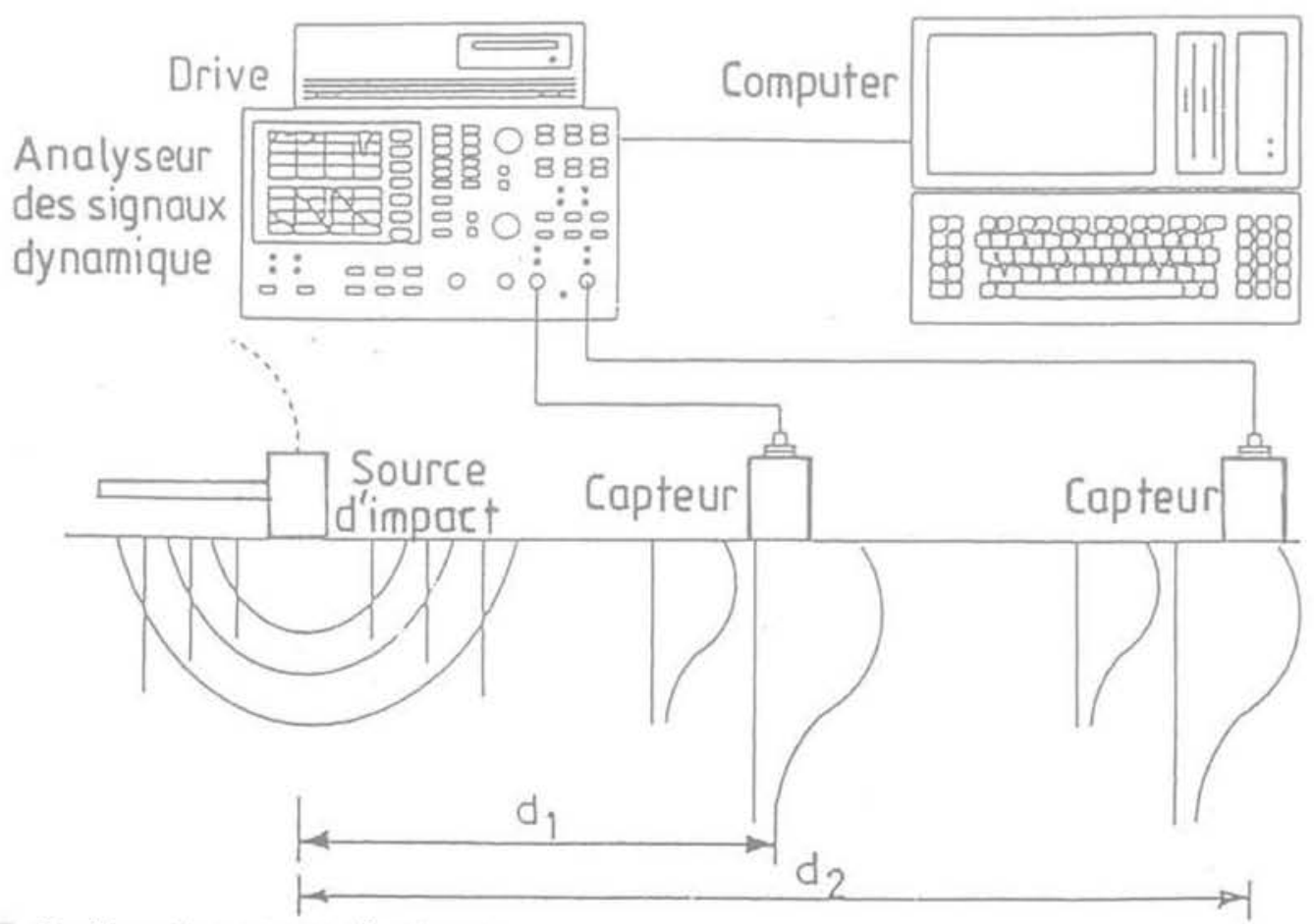

FIG.19 Configuration source-récepteurs.

Source-receiver configuration. 


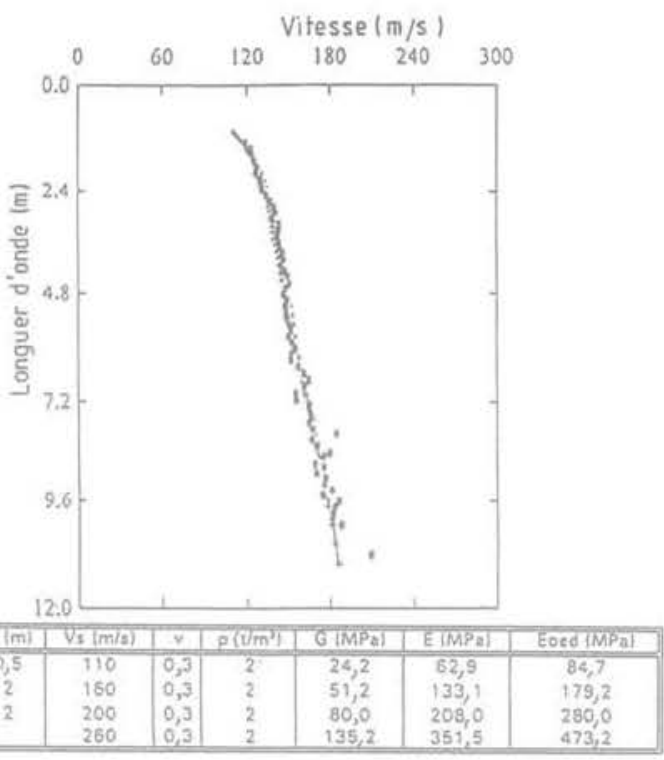

F6. 20 Essai d'analyse spectrale des ondes de surface sur le remblai après ??? (d'après Haegeman and Van Impe, 1995).

Spectral analysis of surface waves on the embankment after placement of fills (after Haegeman and Van Impe, 1995).

\section{Exemple d'application}

Le site étudié est situé près de Gand (Belgique). Il s'agit d'un remblai de sable comme culée de pont. Sa hauteur est de $10 \mathrm{~m}$ et sa longueur de $9 \mathrm{~m}$. La figure 20 montre le résultat d'un essai SASW effectué sur le remblai, l'état de sol étant plus ou moins compact. Cette figure montre un profil typique de sol où la vitesse de l'onde de cisaillement augmente avec la profondeur. Le

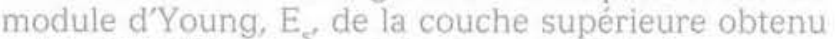
est de 62,9 MPa. Un essai de plaque, effectué au même endroit, a donné une valeur de E, 25,4 MPa. Ce résultat représente approximativement $40 \%$ de la valeur obtenue par la méthode SASW, ce qui est en concordance avec les remarques de Haegeman et Van Impe (1995). En effet, il a été suggéré que, dû aux larges déformations $\left(\sim 10^{-1} \%\right)$ liées avec l'essai à la plaque, le module $E_{p}$ ne représentera que 30 à $40 \%$ du module obtenu par la méthode SASW, valable pour les très petites déformations $\left(10^{-4} \%\right)$.

Un compactage de surface a été effectué quelques semaines après le placement du remblai. Les résultats de l'essai SASW, au même endroit sont montrés sur la figure 21. On peut clairement voir que la vitesse élevée à la surface donne un module de $229,3 \mathrm{MPa}$ et une couche compactée d'épaisseur $0,4 \mathrm{~m}$. On remarquera aussi que, sous cette profondeur, la rigidité décroît. Ceci est dû aux très fortes pluies qui se sont abattues sur la région la veille de l'essai.

L'exemple traité ci-dessus montre qu'on peut distinguer les surfaces compactées grâce à l'amélioration significative de la vitesse de l'onde de cisaillement. Plus important, les valeurs de $\mathrm{V}_{\mathrm{s}}$ peuvent être converties en module de rigidité sans avoir recours à l'empirisme.

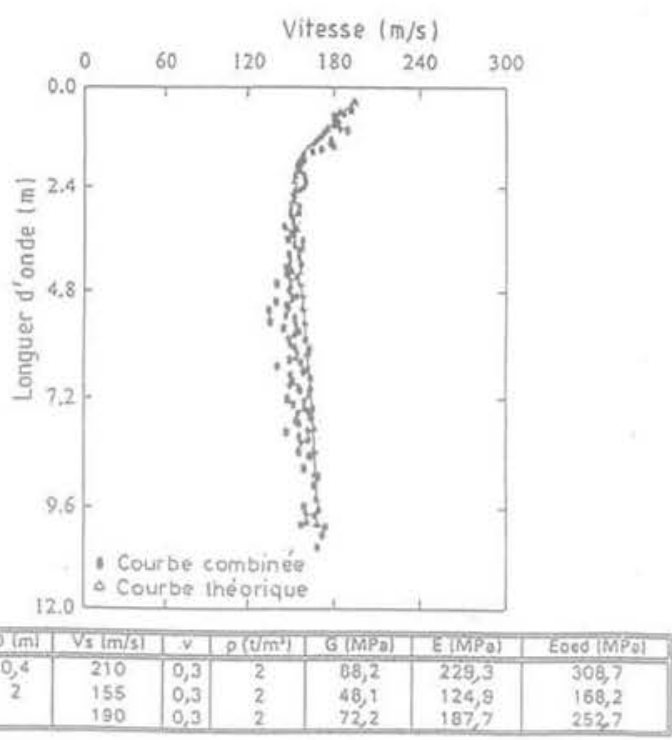

FIG: o1 Essai d'analyse spectrale des ondes de surface sur le remblai après un compactage superficiel (d'après Haegeman et Van Impe, 1995). Spectral analysis of surface waves on the embankment after a shallow compaction fafter Haegeman and Van Impe, 1995).

\section{Conclusion}

Les observations les plus importantes qui peuvent être émises sont:

Les prescriptions réglementaires régissant la conception de dispositifs d'étanchéité varient d'un pays à un autre. Les facteurs qui contribuent à cette variation sont la conscience écologique du public, la volonté politique et les philosophies de gestion de déchets en cours. Les Etats-Unis ont introduit des prescriptions assez strictes. Quelques pays européens seulement ont suivi ce concept.

La barrière d'étanchéité composite pour les décharges de type 1 est recommandée dans la plupart des pays. Cependant, pour les décharges de type 2, des pays comme les États-Unis et l'Autriche proposent des dispositifs d'étanchéité plus élaborés et plus complexes.

Les prescriptions réglementaires ont établi, dans la plupart des cas, une valeur limite de la conductivité hydraulique sans reconnaitre l'effet prédominant de la diffusion. Il est important d'introduire ce facteur dans la réglementation. A notre avis une valeur limite de la conductivité hydraulique est une condition nécessaire, mais pas suffisante.

Le cas des sols non saturés doit être pris en compte pour éviter un calcul très conservatif.

Les recommandations du GLR (ETC8, 1993) ne semblent pas avoir été adoptées par tous les pays européens. A notre avis, le travail fait par l'ETC8 peut être amélioré. Il constitue déjà une bonne base pour une discussion élargie et une harmonisation des différentes prescription réglementaires.

L'épaisseur minimale de la barrière argileuse varie considérablement d'un pays à un autre. Dans ce cas, le 
concepteur doit utiliser son " expérience ou jugement d'ingénieur m) et ne pas se restreindre seulement aux "critères standards» pour assurer une performance satisfaisante.

Le géocomposite est un matériau nouveau prometteur. Cependant, son utilisation doit être faite avec précaution, car il nécessite une main-d'cuvre qualifiée pour éviter des problèmes sur site. Son comportement à long terme dans un milieu agressif est encore mal connu,

Le choix des géomembranes est dicté par le type de déchets à stocker; leur comportement à très long terme restent encore un sujet de discussion.

Le contrôle de la qualité du compactage est un facteur essentiel dans la construction de la barrière argileuse. La méthode non intrusive de l'analyse spectrale des ondes de surface permet un contrôle méticuleux et rigoureux des surfaces compactées sans risque de les détériorer.

Enfin, d'autres facteurs tels que le frottement géosynthétique/sol, stabilité des pentes de la décharge, etc., n'ont pas été abordés dans ce texte. Ils jouent aussi un rôle important dans la conception du dispositif d'êtanchéité d'un site de décharges.

\section{Remerciements}

Le second auteur remercie le NFWO et le Laboratoire de Mécanique des sols de l'Université de Gand pour le support scientifique, l'aide financière et matérielle fournis dans le cadre de ce travail.

\section{Bibliographie}

Addo K.O., Robertson P.K. - Shear wave velocity measurement of soils using Rayleigh waves. Canadian Geotechnical Journal, vol. 29, 1992, p.558-568.

Amman P.. Martinenghi L. - Geotechnical design criteria for landfills and waste disposal sites, Joint CSCE-ASCE, National Conference on Environmental Enqineering, Montreal (Canada), 1993 (offprint).

Anon - La Russie meurt de sa pollution. Le Soir, 29/04/95, p. 25.

Barbosa C., De Almeida M., Ehrlick M. Mécanismes de transporte de contaminants em Una argila organo salina da bara de Guanabaran, R.J., Proc. LusoBrazilian Environmental Geotechnic Conference, Lisbon (Portugal), 1995.

Bennett G.F. - Air quality aspects of hazardous waste landfills. Journal of Hazardous Waste and Hazardous Material, vol. 4. $n^{\circ} 2,1987$, p. 119

Benson C.H., Daniel D.E. - Minimum thickness of compacted soil liners, II. Stochastic models. Journal of Geotechnical Engineering, vol. $120, n^{\circ} 1.1994 a$, p. 129-152.

Benson C.H. Daniel D.E. - Minimum thickness of compacted soil liners, II. Analysis and case histories, Journal of Geotechnical Engineering, vol. 120, $n^{\circ} 1$. 1994a, p. 129-152

Bishop D.J, Cartier G. - Waste disposal by landfill-German landfill lining systems, Proc. Int. Symp. on Geotechnics Related to the Environment (Green 93), Bolton (UK), 1993, p. 127-134.

Bonaparte R., Goss B.A. - Field behaviour of double liner systems, Proc. Waste Containment Systems, GSP n ${ }^{\circ} 26$, ASCE, San Francisco (USA), 1990, p. 52-72.

Bonaparte R. - Long term performance of landfilis, Geoenvironment 2000, GSP $n^{\circ} 46$, ASCE, New Orleans (USA), 1995. vol. 1, p. 514-553.

Bouazza A. - The geotechnical engineer and the environment protection in Algeria, Proc. Int. Conf. Environment and Geotechnic, Paris (France), 1993, p. 153-157.

Bouazza A. - Compacted clayed soils as cover material in landfills, Proc. Int. Symp. on Geotechnics Related to the Environment (Green 93), Bolton (UK), 1993a, p. 405-407.

Brandl H. - Mineral liners for hazardous waste containment. Geotechnique, vol. $42, n^{\circ} 1,1992$, p. $57-65$.
Come B. - Contributions dé la géologie de l'ingénieur au stockage surr des dèchets mènagers et industriels toxiques: exemples actuels et perspectives, Proc. 7th int. IAEG Congress. Lisbon (Portugal), vol. IV, 1994, p. XVIIXXXVII.

Conca J.-L., Wright J. - Diffusion coefficient in gravel under unsaturated conditions. Water Resour. Res, vol. $26, n^{\circ} 5$. 1990, p. 1055-1066.

Crank J, - The mathematics of diffusion. Clarendon Press, Oxford, 1975, 414 p.

Crooks V.E., Quigley R.M. - Saline leachate migration through clay: A comparative laboratory and field investigation. Canadian Geotechnical Journal, vol, 21, 1989 p. 349-362.

Daniel D.E. - Pollution prevention in landfills using engineered final covers, Proc Int. Symp. on Geotechnics Related to the Environment (Green 93), Bolton (UK), 1993, p. 73-92.

Doll P., Wessolek G. - Bodenphysikalische Eigenschaften und temperatur-abhängiger Wasserhaushalt einer Tonabdichtung, Proc. 10 Nurrnberger Deoponieseminar, Veroffentlichungen des LGA-Grundbatinstituts, Nürnberg, 1994.

Estornell P., Daniel D.E. - Hydraulic conductivity of three geosynthetic clay liners. Journal of Geotechnical Encineering. vol. $118, \mathrm{n}^{\circ} 10,1992$, p. 1592 1606.

ETC 8 - Geotechnics of landfill design and remedial works technical recommendations-GLR, Ernst \& Sohn (publ.), 1993.

Freeze R.A., Cherry J.A. - Groundwater. Prentice Hall, Englewood Cliffs, N.J. 1979, 604 p.

Gillham R.W., Robin M.L.J., Dytynyshyn D.J., Johnston H.M. - Diffusion of non reactive and reactive solutes through fine grained barrier materials. Canadian Geotechnical Journal, vol.21, 1984, p.541-550.

Giroud J.P., Bonaparte R. - Leakage through liners constructed with geomembranes. Part II. Composite liners. Geotextiles \& Geomembranes, vol. 8, $n^{\circ} 2,1989$, p. $78-111$.

Giroud J.P., Cazzuffi D.A. - Uses of geosynthetics for environmental control, Proc. 12th Int. Conf. SMFE Rio de Janeiro (Brasil), vol. 5, 1989, p. 3119-3125.
Goodall D.C.. Quigley R.M. - Polluant migration from two sanitary landfill sites near Sarnia, Ontario. Canadian Geotech. Journal, vol. 14, 1977, p. 223. 236.

Gordon M.E., Huebner P.M., Mitchell G.R. - Regulation, construction and performance of clay lined landfills in Wisconsin, Proc. Waste Containment systems, GSP n ${ }^{\circ}$ 26, San Francisco (USA), 1990, p. $14-29$.

Haegeman W.. Van Impe W.F. - More reliable soil liquefaction characterisation and soil deformation behaviour by SASW, Proc Seminar Soil Mechanics and Foundation Engineering, Sigtune (Sweden). 1993.

Haegeman W., Van Impe W.F, - Evaluation of heavy tamping on a waste dis. posal by the SASW method, Proc X European Conf. Soil Mechanics and Foundation Engineering. Copenhagen (Denmark), vol. 2, 1995, p. 133-140.

Haegeman $W_{-}-$Non destructive evaluation of a road construction by the SASW method, Danube Conf. on Soil Mechanics and Foundation Engineering, Bucarest (Romania), 1995 (sous presse).

Fahim A. Koerner R.M. - A survey of state municipal solid waste liner and cover systems, GRI report, \# 11. Drexel University, 1993

Jessberger H.L. - Waste containment with compacted clav liners, Geoenvi ronment 2000, GSP $n^{\circ} 46$, vol. 1, 1995, p. $463-483$.

Johnson R.I.. Cherry J.A.. Pankow J.F. Diffusive contaminant transport in natural clays: A field example and implication for clay-lined waste disposal sites Environ. Sci Technology, vol. 23, 1989, p. $340-349$.

Jost W. - Diffusion in solids, liquides and Gases, Academic Dress, N.Y.. 1960, $558 \mathrm{p}$.

Kavazanjian E., Snow M.S., Matasovic N., Poran C.J., Satoh T. - Non intrusive Rayleigh wave investigations at solid waste landfills, Proc. Int. Congress Environ. Geotechnics, Edmonton (Canada), 1994, p. $707-712$.

Keenan J.D. - Landfill leachate management Journal of Resource management and technology, vol. $14, n^{\circ} 3,1986$, p. 177-180. 
Koerner R.M., Hsnau Y., Lord jr A.E. Remaining technical barriers to obtain general acceptance of geosynthetics. Grouting. Soil Improvernent and Geosynthetics, GSI. n³0, ASCE, vol. 1. 1992, p. 63-109.

Kovacic D., Mayer D., Muhovec L. - Geotechnical characteristics of Zagreb waste disposal site and possibilities of it reclamation, Proc. Int. Symp. on Geotechnics Related to the Environment (Green 93), Bolton (UK), 1993, p. 543-547.

Madsen F., Nuesch R. - Characteristics and sealing effect of bentonites, Proc Int. Symp. Geosynthetics clay liner. Nurenberg (Germany), 1994, p. 31-50

Mancuso C Vinale. F - Use of SASW in earthdam investigation, Proc. Int. Conf. Geotechn. Engineering, Hard Soils-Soft Rocks, Athenes (Greece), vol. 2, 1993 , p. 1291-1298.

Mitchell J.K. - Conduction phenomeno from theory to Geotechnical practice. Geotechnique, vol. 41, n³, 1991, p. 299 340.

Muurinene A. - Diffusion of uranium in compacted sodium bentonite. Eng. geology, vol. 28, 1990, pp. 359-367, n²6, San Francisco (USA), p. 156-174.

Nazarian S. Stokoe K.H - In situ shear wave velocities from spectral analysis of surface waves. Proc. 8th World Conf. Earthquake Eng. San Francisco (USA). vol. 3, 1984, p. 31-38.

Nazarian S. - In situ determination of elas tic moduli of soil deposits and pavement systems by SASW method, PhD thesis, University of Texas at Austin (USA), 1984.
Noorany I. - Variability in compaction Control. Journal of Geotechnical Engineering, vol. 116, $n^{\circ} 7,1990, \mathrm{p} .1132-1136$.

Pohland F.G. Harper S. R. - Critical review and summary of leachate and gas production from landfills, Georgia Institute of Technology, 1985.

Potter H.A.B., Yong R.N. - Waste disposal by landfill in Britain: problems, solutions, and the way forward. Proc. Int. Symp. on Geotechnics Related to the Environment (Green 93), Bolton (UK). 1993, p. 167-173.

Reades D.W., Lahti L., Quigley R.M. Bacoupolos A. - Detailed case history of clay liner performance, Proc. Waste Containment Systems, GSP n ${ }^{\circ} 26$, San Fransisco (USA), 1990, p. 156-174.

Rebeiz K.S., Mielich K.L. - Construction use of municipal solid waste ash. Journal of Energy Engineering, vol.121, $1^{\circ} 1$, 1995, p. 2-13.

Rowe R.K. - Polluant transport through barriers, Geotechnical Practice for waste disposal, GSP n ${ }^{\circ} 13$, ASCE, 1987, p. 159-191.

Rowe R.K. - Some considerations in the design of barrier systems. Proc. 1st Canadian Conf. on Environmental Geotechnics, Montreal (Canada), 1991. p. 157-164.

Scherbeck - Geotechnisches Verhalten Mineralischer Deponieabdichtungen beiungleichförm Verformungseinwirkung, Schriftenreihe des Instituts für Grundbau, Ruhr-Universität Bochürr. 1992.

Shackelford C.D. - Transit time design of earthen barriers. Engineering Geology, vol. 29, 1990, p. 79-94.
Shackelford C.D. - Laboratory diffusion testing for waste disposal. A review. Journal of contaminant Hydrology, vol.7. 1991, p. 177-217.

Shackelford C.D. - Waste-soil interactions that alter hydraulic conductivity, ASTM STP 1142, Hydraulic conductivity and waste contaminant transport in soil, 1994, p. 111-168.

Sills B.. Mitchell R. - A new method for studying diffusion in unsaturated soil, Geoenvironment 2000, GSP $\mathrm{n}^{\circ} 46$, ASCE, vol. 1, 1995, p. 346-354.

Street A. - Landfilling: the difference between continental European and British practice, Proc. Inst. Civ. Engineers, Geotechnical Engineering, vol.107, 1994. p. 41-46.

Thomas H., Jensen D., Authier B. - Remediation of crude-oil-contaminated soils beneath a containment liner, Geoenvironment 2000, GSP n ${ }^{\circ} 46, \mathrm{ASCE}$, vol. 2 . 1995, p. 1506-1517.

Van der Poel C. - Dynamic testing of road constructions. Journal App. Chem., vol. 1. 1951, p. 7 .

Van Genutchen M.T.A. Parker 3.C. Boundary conditions for displacement experiments through short laboratory soil columns. Journal Soil Science, Soc. of America, vol. 48, 1984, p. 703-708.

Van Impe W.F., Bouazza A. - General comments on liner design for waste disposal sites, Proc. Luso-Brazilian Conf, in Environ. Geotechnics, Lisbon (Portugal), 1995.

Van Impe W.F. - Cours de la géotechnique de l'environnement, Univ, de Gand, 1995 (en anglais). 